\title{
A Tale of Seemingly “Identical” Silicon Quantum Dot Families: Structural Insight into Silicon Quantum Dot Photoluminescence
}

Alyxandra Thiessen, Lijuan Zhang, Anton Oliynyk, Haoyang Yu, Kevin O'Connor, Alkiviathes Meldrum, Jonathan G.C. Veinot

Submitted date: 14/02/2020 - Posted date: 17/02/2020

Licence: CC BY-NC-ND 4.0

Citation information: Thiessen, Alyxandra; Zhang, Lijuan; Oliynyk, Anton; Yu, Haoyang; O'Connor, Kevin; Meldrum, Alkiviathes; et al. (2020): A Tale of Seemingly “Identical” Silicon Quantum Dot Families: Structural Insight into Silicon Quantum Dot Photoluminescence. ChemRxiv. Preprint.

https://doi.org/10.26434/chemrxiv.11852040.v2

Two quantum dots, both alike in composition, but differing in structure, where we lay our scene. From broader classes, to bring deeper understanding, to the crystalline core that drives the quantum dot's sheen. In this contribution we examine two families of silicon quantum dots (SiQDs) that bring to mind the Capulets and the Montagues in Shakespeare's Romeo and Juliet because of their stark similarities and differences. SiQDs are highly luminescent, heavy-metal-free and based upon earth-abundant elements. As such, they have attracted attention for far reaching applications ranging from biological imaging to luminescent solar concentrators to light-emitting diodes that rely on their size-dependent optical response. Unfortunately, correlating SiQD "size" to their photoluminescence maximum is often challenging. Herein, we provide essential structural insight into the correlation of SiQD dimension and PL maximum through a direct comparison of samples that exhibit statistically identical physical dimensions $\left(\mathrm{d}_{\mathrm{TEM}}\right)$ and chemical compositions, but different crystallite size $\left(d_{X R D}\right)$ and PL maxima. We then expand the scope of this investigation and systematically compare groupings of SiQDs: one in which the $\mathrm{d}_{X R D}$ and $\mathrm{d}_{\mathrm{TEM}}$ agree and one where $\mathrm{d}_{\mathrm{XRD}}<\mathrm{d}_{\mathrm{TEM}}$. This latter comparison clearly shows $d_{X R D}$ better predicts SiQD optical response when using the well-established effective mass approximation.

File list (2)

Two_Families_of_SiQDs.pdf (710.18 KiB) view on ChemRxiv - download file 


\section{A Tale of Seemingly "Identical” Silicon Quantum}

2 Dot Families: Structural Insight into Silicon

3 Quantum Dot Photoluminescence.

4

5 Alyxandra N. Thiessen ${ }^{a}$ Lijuan Zhang $^{b}$, Anton O. Oliynyk ${ }^{c}$, Haoyang Yu ${ }^{a}$, Kevin M. O'Connor ${ }^{a}$, Alkiviathes Meldrum ${ }^{b}$, and Jonathan G. C. Veinot ${ }^{a^{*}}$

${ }^{b}$ Department of Physics, University of Alberta, Edmonton, Alberta T6G 2E1, Canada 


\section{Abstract}

2 Two quantum dots, both alike in composition, but differing in structure, where we lay our scene.

3 From broader classes, to bring deeper understanding, to the crystalline core that drives the quantum

4 dot's sheen. In this contribution we examine two families of silicon quantum dots (SiQDs) that

5 bring to mind the Capulets and the Montagues in Shakespeare's Romeo and Juliet because of their

6 stark similarities and differences. SiQDs are highly luminescent, heavy-metal-free and based upon

7 earth-abundant elements. As such, they have attracted attention for far reaching applications

8 ranging from biological imaging to luminescent solar concentrators to light-emitting diodes that

9 rely on their size-dependent optical response. Unfortunately, correlating SiQD "size" to their

10 photoluminescence maximum is often challenging. Herein, we provide essential structural insight

11 into the correlation of SiQD dimension and PL maximum through a direct comparison of samples

12 that exhibit statistically identical physical dimensions ( $\left.\mathrm{d}_{\mathrm{TEM}}\right)$ and chemical compositions, but

13 different crystallite size ( $\left.\mathrm{d}_{\mathrm{XRD}}\right)$ and PL maxima. We then expand the scope of this investigation

14 and systematically compare groupings of SiQDs: one in which the $\mathrm{dXRD}_{\mathrm{X} D}$ and $\mathrm{d}_{\mathrm{TEM}}$ agree and one

15 where $\mathrm{d}_{\mathrm{XRD}}<\mathrm{d}_{\mathrm{TEM}}$. This latter comparison clearly shows $\mathrm{d}_{\mathrm{XRD}}$ better predicts SiQD optical 16 response when using the well-established effective mass approximation.

17 KEYWORDS: Quantum dots; Silicon nanocrystals; Silicon; Luminescence; Lifetimes 
1 Semiconductor nanoparticles (quantum dots; QDs) are fascinating structures that exhibit size- and

2 shape-dependent optoelectronic properties. Prototypical CdSe@ZnSQDs have been widely

3 studied, are generally well understood, and many reports of prototype applications have appeared;

4 in fact, InP-based QDs are revolutionizing consumer electronics as active systems in QLED-TVTM

5 displays. ${ }^{1,2}$ Unfortunately, legislation limits use of heavy metals in consumer products and curtails

6 widespread implementation of many QDs; ${ }^{3}$ furthermore, alternative QD materials such as InP

7 contain costly non-earth abundant elements and exhibit 'clear evidence of carcinogenic activity'

8 in animal models. ${ }^{4}$ In this context, QDs comprised of abundant, non-toxic elements must be

9 prepared, manipulated, understood, and deployed. ${ }^{5}$

10 Silicon-based QDs are attractive for many applications because they are heavy metal-free, 11 comprised of earth-abundant elements, and biologically compatible. In this context, a variety of

12 methods have been developed to prepare and functionalize these promising materials ${ }^{6-11}$ and 13 prototype applications including light-emitting diodes, ${ }^{12}$ luminescent solar concentrators 14 (LSCs), ${ }^{13}, 14$ biological imaging agents, $,{ }^{15}, 16$ sensors, ${ }^{17,} 18$ and lithium ion battery anodes ${ }^{19,}{ }^{20}$ have

15 appeared. Many of these uses rely on the unique tailorable optical properties of SiQDs; for example, LSCs take advantage of the large Stokes shift (i.e., the energy difference between

17 excitation and photoluminescence maxima) that arises in part because of the indirect nature of the

18 Si band gap. ${ }^{13,14}$ Despite impressive advances including spectral tuning throughout the full visible

19 region and photoluminescence (PL) quantum yields rivaling that of compound semiconductor 20 QDs, challenges remain; ${ }^{21-25}$ paramount among these is the limited predictability of the PL 21 maximum size dependence that appears throughout the literature. ${ }^{26}$

22 The origin of SiQD PL is clearly complex; ${ }^{27-32}$ for convenience, SiQDs can be categorized into 23 two broad groupings based upon PL properties and associated excited-state lifetimes. ${ }^{28}$ The first 
1 involves SiQDs exhibiting PL characterized by short-lived excited states (i.e., $\tau=$ nanoseconds);

2 in these cases the PL maximum that often appears in the blue spectral region (i.e., 390-500 nm), is

3 dependent on excitation wavelength, and largely independent of particle size. Such behavior has

4 previously been attributed to surface-state mediated relaxation processes involving heteroatom

5 containing species (e.g., $\mathrm{N}, \mathrm{Cl}$, O, etc.). ${ }^{21,} 28,29,33-35$ Other SiQDs show PL throughout the

6 yellow/orange to near-IR regions with long-lived excited states (i.e., $\tau=$ microseconds) consistent

7 with the indirect band gap of silicon; the proposal that this luminescence arises from band gap-

8 based processes is further supported as the PL maximum generally trends with particle size and is

9 broadly consistent with carrier quantum confinement. ${ }^{25,29,33,36}$

10 A comprehensive review of SiQDs exhibiting microsecond lifetimes has appeared and a detailed

11 discussion is beyond the scope of the present contribution. ${ }^{33}$ However, for context it is useful to

12 consider that while the PL response of these QDs has been attributed to carrier confinement, the

13 PL maximum of seemingly identical particles can vary greatly and its energy frequently does not

14 correlate well with effective mass approximation (EMA) predictions (Figure 1). ${ }^{26,37-39}$ Numerous

15 explanations for these deviations have appeared; broad particle size distributions, ${ }^{40}$ among

16 others. ${ }^{38,41}$ As such, alternative strategies (e.g., empirical pseudopotential approximation (linear

17 combination of atomic orbitals), ${ }^{42,}{ }^{43}$ empirical tight binding band theory, ${ }^{44}, 45$ ab-initio local

18 density approximation $)^{46}$ for correlating PL maximum to SiQD dimensions have been proposed. ${ }^{26 \text {, }}$ 


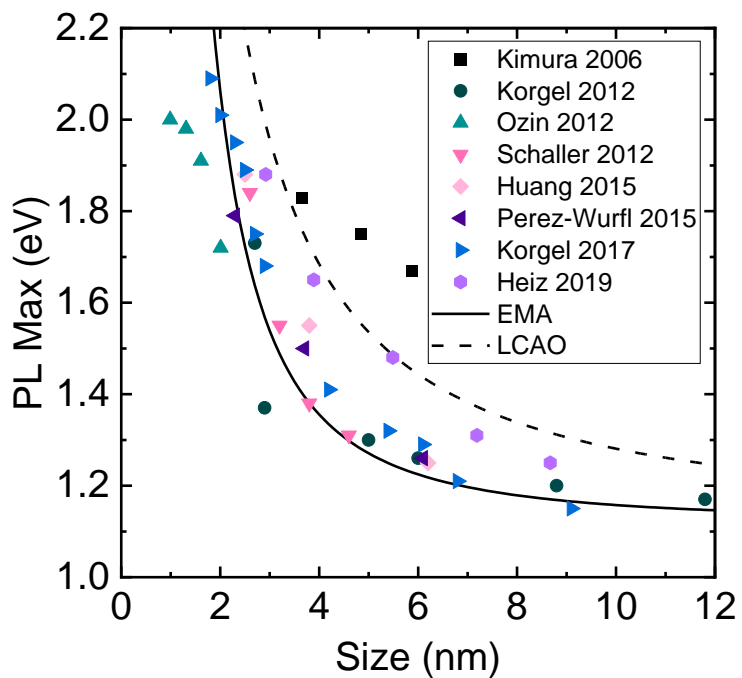

2 Figure 1: A summary representative literature data showing the relationship between SiQD peak

3 PL emission and particle dimension. ${ }^{37,39,40,47-51}$ Predicted relationships obtained using the

4 effective mass approximation ${ }^{52}$ (solid line; $E_{g}(r)=1.12+3.77 / r^{2}$ ) and linear combination of

$5 \quad$ atomic orbitals ${ }^{26,42}$ (dashed line; $E_{g}(r)=1.12+3.77 / r^{1.39}$ ) are shown for comparison.

6 It is well-established that many challenges (as well as intriguing properties) associated with

7 preparing and exploiting SiQDs arise from the extended covalent bonding manifested within the

8 Si diamond-like crystal structure. While amorphous surface layers have been implicated in ultra-

9 fast SiQD photoluminescence, ${ }^{53-56}$ the uniformity of the SiQD internal structure has largely been

10 overlooked. ${ }^{57}$ We previously demonstrated the Si core of large $\left(\mathrm{d}_{\mathrm{TEM}} \sim 9-64 \mathrm{~nm}\right)$ SiQDs exhibits

11 a radially graded structure consisting of a highly disordered surface that transitions to a crystalline

12 core; in contrast, the structure of small SiQDs $\left(\mathrm{d}_{\mathrm{TEM}} \sim 3 \mathrm{~nm}\right)$ is dominated by disorder and they

13 only possess very small crystallite regions (i.e., $\mathrm{d}_{\mathrm{XRD}} \sim 1.2 \mathrm{~nm}$ ) ${ }^{57}$ Knowing the electronic structure

14 of bulk silicon strongly depends on crystallinity (e.g., amorphous Si Eg,optical= 1.6-1.7 eV; bulk

15 crystalline $\left.\mathrm{Si} \mathrm{E}_{\mathrm{g}}=1.1 \mathrm{eV}\right),{ }^{58}$ we endeavored to explore the impact of internal structure on SiQD

optical response. Herein, we describe an evaluation of the size dependence of SiQD PL maximum 
1 and excited-state lifetime while systematically varying particle and crystallite sizes as determined

2 by transmission electron microscopy (TEM) and X-ray powder diffraction (XRD), respectively.

\section{Results and discussion}

4 To probe the impact of an internally graded crystal structure on SiQD optical properties it was

5 necessary to predictably prepare QDs with the same particle (determined using TEM; $\mathrm{d}_{\text {TEM}}$ ) and

6 different crystallite (determined using XRD, $\mathrm{d}_{\mathrm{XRD}}$ ) sizes while maintaining all other contributing

7 factors (e.g., surface chemistry/oxidation) the same. Drawing on our intimate knowledge of the

8 hydrogen silsesquioxane (HSQ) -based procedure and the structure of the resulting SiQDs, ${ }^{57,59-61}$

9 we prepared two different composites consisting of nanocrystalline silicon domains encased within

10 an $\mathrm{SiO}_{2}$-like matrix via $\mathrm{HSQ}$ reductive thermal processing at 1200 and $1300{ }^{\circ} \mathrm{C}$. Evaluation of the

11 Si domain sizes using TEM was precluded by the oxide matrix however, consistent with

12 expectation, ${ }^{37,60} \mathrm{XRD}$ revealed reflections attributable to nanocrystalline Si domains and that, as

13 expected, larger domains are obtained for products processed at higher-T (i.e., $\mathrm{d}_{1200 \sim} 3.7 \mathrm{~nm}$;

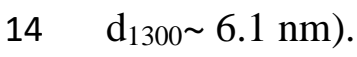

15 The nanocrystalline domains were liberated as freestanding SiQDs upon etching with alcoholic 16 aqueous hydrofluoric acid. Drawing on the expectation that amorphous/disordered silicon is more 17 susceptible to this etching process, careful definition of the etching time allowed isolation of 18 SiQDs from the two composites that possess statistically identical $\mathrm{d}_{\text {TEM }}$ but different $\mathrm{d}_{\mathrm{XRD}}$ 19 dimensions (i.e., different thicknesses of disordered Si shells). Following isolation, the SiQD 20 surfaces were functionalized with dodecyl- moieties (confirmed to be identical at the sensitivity of 21 the FTIR technique; Figure S1) using size independent radical-induced hydrosilylation to render 22 them solution processable and minimize reactions (e.g., oxidation) that are known to impact optical 
1 response. ${ }^{11}$ For the following discussion, SiQDs resulting from composites prepared at 1200 and

$2 \quad 1300{ }^{\circ} \mathrm{C}$ will be referred to as $1200-\mathrm{SiQD}$ and $1300-\mathrm{SiQD}$, respectively.

3 Prior to evaluating the size and optical properties of the SiQDs, it is essential to confirm the degree

4 and speciation of surface oxidation is consistent across the samples because these factors can also

5 impact SiQD PL response. ${ }^{29,62}$ To do so, the Si 2p XP spectra of the 1200- and 1300-SiQDs

6 (Figure 2a) were deconvoluted into component spin-orbit couples ( $\mathrm{Si} 2 \mathrm{p}_{1 / 2}$ and $2 \mathrm{p}_{3 / 2}$ )

7 corresponding to $\mathrm{Si}(0)$ (i.e., orange), $\mathrm{Si}(\mathrm{I})$ (i.e., green), and $\mathrm{Si}(\mathrm{II})$ (i.e., blue). ${ }^{57}$ Integrating the total

8 area of the $\mathrm{Si}$ (I) and $\mathrm{Si}(\mathrm{II})$ components and comparing it to the integrated area of the $\mathrm{Si}(0)$

9 component, provided an estimation of the ratio of oxidized surface silicon species to elemental

10 silicon. For the present samples, the total integrated signal of the $\mathrm{Si}(\mathrm{I})$ and $\mathrm{Si}(\mathrm{II})$ components

11 corresponds to $21 \%$ for $1300-\mathrm{SiQDs}$ and $27 \%$ for $1200-\mathrm{SiQDs}$ of the total Si $2 \mathrm{p}$ signal intensity.

12 This, combined with the reasonable assumption that the degree of surface functionalization (i.e.,

13 surface density of Si-C bonds) is equivalent for both samples indicates 1200- and 1300-SiQDs

14 have similar amounts of oxidation. Furthermore, closer inspection of the $\mathrm{O}$ and $\mathrm{Si}$ spectral

15 envelopes indicates the speciation of the oxides is near identical. In this context, we expect the

16 influence (if any) of surface oxide species on the optical properties of 1200- and 1300-SiQDs to

17 be equivalent.

18 Turning to the evaluation of QD particle and crystallite sizes, bright-field TEM analyses of the

19 liberated dodecyl-functionalized particles afforded $\mathrm{d}_{\text {TEM }}$ of $5.3 \pm 1.4$ and $5.4 \pm 1.1 \mathrm{~nm}$ (Figure $2 \mathrm{~b}$ )

20 for the 1200- and 1300-SiQDs, respectively, and are statistically similar to a $95 \%$ confidence

21 interval. To complement the TEM analyses, crystallite sizes of the identical samples were

22 determined using XRD. Diffraction data were fit to the NIST Si line shape standard (640f) as an

23 "infinite" crystallite reference to account for instrumental broadening and line shape. Having 
1 accounted for instrumental factors, Gaussian and Lorentzian peak shape parameters, with the

2 assumption that atomic displacement parameters and crystallographic site deficiencies do not play

3 a role, only size and strain contributions to line broadening remain and can be qualitatively

4 accounted for based on their diffraction angle dependence. ${ }^{57,63}$ In contrast to the $\mathrm{d}_{\mathrm{TEM}}$ which were

5 statistically identical, the $\mathrm{d}_{\mathrm{XRD}}$ (i.e., crystallite sizes) determined from diffraction data (Figures 2e

6 and $2 \mathrm{f}$ ) were $3.2 \pm 1.22$ and $4.7 \pm 0.4 \mathrm{~nm}$ for 1200-SiQDs and 1300-SiQDs, respectively. A

7 straightforward comparison of the $\mathrm{d}_{\mathrm{TEM}}$ and $\mathrm{d}_{\mathrm{XRD}}$ clearly shows that, despite having the same

8 physical dimensions, 1200-SiQDs have a smaller crystallite size (and by extension thicker

9 disordered $\mathrm{Si}$ shell). This is further supported by high annular angle dark field scanning

10 transmission electron microscopy (HAADF-STEM) that shows thicker disordered layers on 1200-

11 SiQDs (1.7 nm 1200-SiQDs vs. $1.0 \mathrm{~nm}$ for 1300-SiQDs; Figure S2 and S3).

12 
(a)
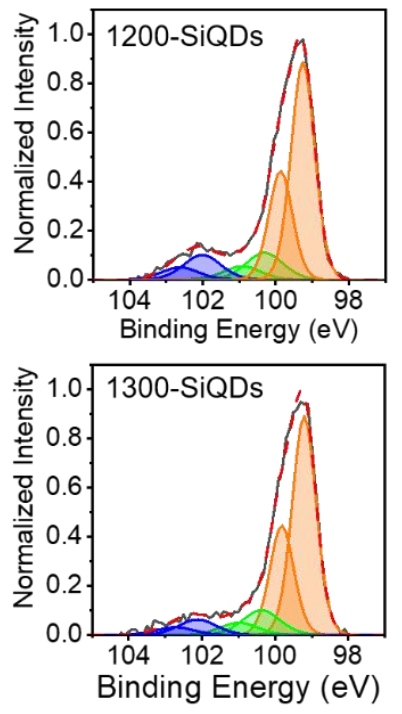

(b)
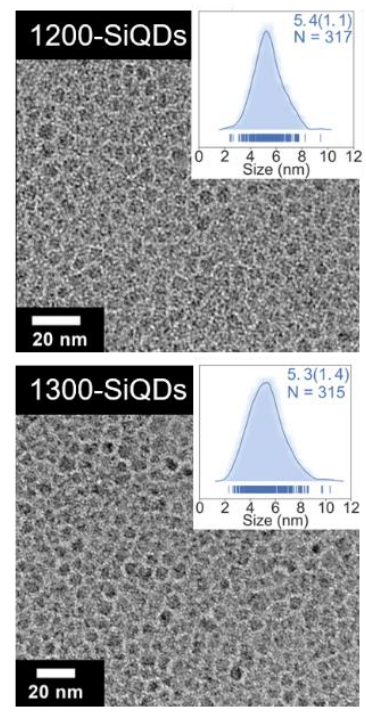

(c)

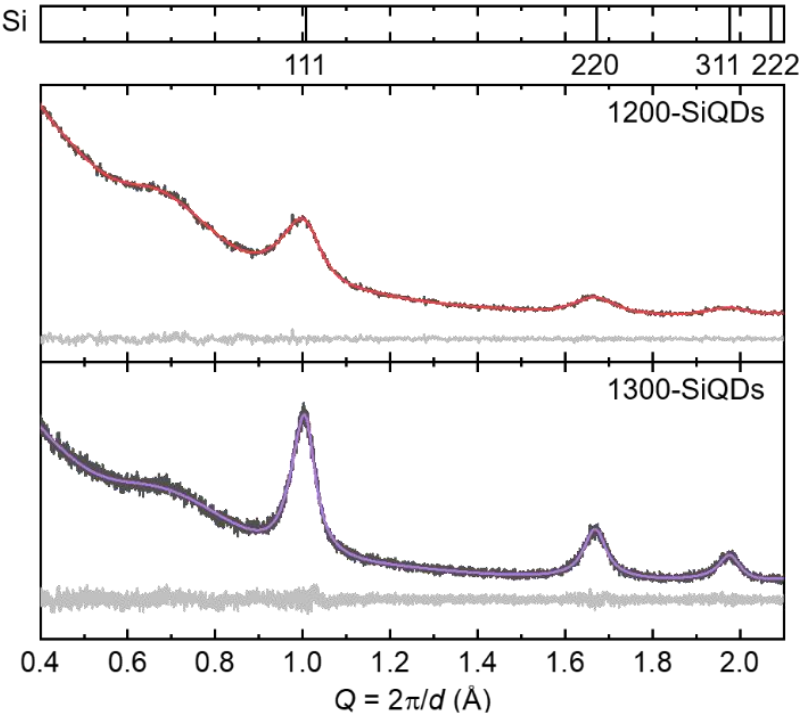

2 Figure 2: a) Si 2p XP spectrum with deconvolution/fitting of the data for 1200-SiQDs and 1300-

3 SiQDs. Fitting peaks are shown as $\mathrm{Si} 2 \mathrm{p}_{1 / 2}$ and $2 \mathrm{p}_{3 / 2}$ components corresponding to $\mathrm{Si}(0)$ (i.e.,

4 orange), $\mathrm{Si}(\mathrm{I})$ (i.e., green), and $\mathrm{Si}(\mathrm{II})$ (i.e., blue). The black trace is the experimental spectrum

5 and the red dashed line represents the overall fitting envelope. b) TEM image of 1200-SiQDs and

6 1300-SiQDs inset: average shifted histogram with the average size and the distribution width. c)

7 XRD data for 1200-SiQDs and 1300-SiQDs showing experimental powder pattern (black) with

8 fitting (red for 1300-SiQDs and purple for 1200-SiQDs) and residuals (light grey). 
(a)

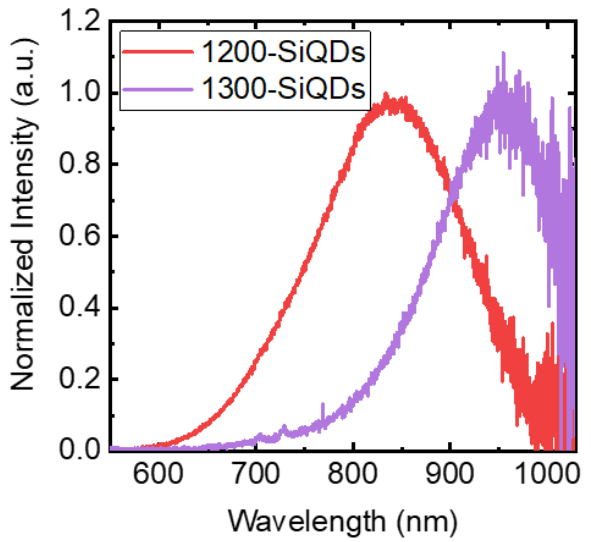

(b)

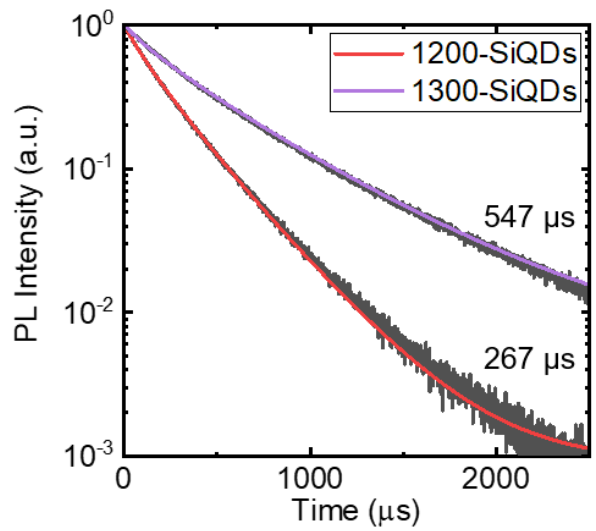

2 Figure 3: Comparing a) the photoluminescence emission of 1300-SiQDs (purple) and 1200-

3 SiQDs (red) as well as b) the luminescence lifetimes of 1300-SiQDs (purple fit) and 1200-SiQDs

4 (red fit). Luminescence lifetimes were fit to a stretched exponential function and the mean

5 lifetimes were then calculated as described in the supplemental information. ${ }^{64}$ The fitting

6 parameters are reported in Table $\mathrm{S} 2$.

7 Having shown 1200-SiQDs and 1300-SiQDs exhibit near-identical physical size (i.e., d dEM) as

8 well as composition (i.e., degree of oxidation, oxidation speciation, surface functionalization) and

9 they only differ in crystallite size (i.e., dXRD), we endeavored to evaluate the impact of the 10 disordered Si shell on the 1200-SiQD optical properties. The PL spectra of SiQD toluene solutions

11 (Fig. 3a) were evaluated using the 351 and $364 \mathrm{~nm}$ lines of an Ar ion laser and show maxima of 
1837 and $955 \mathrm{~nm}$ for 1200-SiQDs and 1300-SiQDs, respectively. The marked red-shift in PL

2 maximum with increased $\mathrm{d}_{\mathrm{XRD}}$ indicates the dimensions of the nanocrystalline domain dominate

$3 \mathrm{SiQD}$ PL and band gap; there is little (or no) indication of a contribution from the disordered

4 surface silicon species to these properties. This observation is also consistent with the amorphous

5 silicon shell having a wider band gap than the crystalline core providing a core@ shell structure

6 similar to that of more commonly studied CdSe@ZnTe systems (i.e., Type II QDs). ${ }^{65}$ Contrary to

7 the CdSe@ZnTe QD case, in which the ZnTe shell forms epitaxially on the CdSe to reduce

8 interfacial dark defects and increase PL QYs, the interface between the crystalline and amorphous

9 components of the present SiQDs can reasonably be expected to be ill-defined. ${ }^{57,66,67}$ As such,

10 there is no obvious trend in the present PL QY data (Figure S5). We also note that 1300-SiQDs

11 exhibit longer PL lifetimes (i.e., $547 \mu$ s for 1300-SiQDs vs. $267 \mu$ s for 1200-SiQDs), which are

12 often noted for larger SiQDs. ${ }^{48}$

13 To further explore the broader scope of the relationship between SiQD optical properties with $14 \mathrm{~d}_{\mathrm{XRD}}, \mathrm{SiQD} / \mathrm{SiO}_{2}$ composites were prepared via reductive thermal processing of HSQ at 1100 , 151200 , and $1300^{\circ} \mathrm{C}$; it is well-established that larger Si nanodomains form at higher temperatures. ${ }^{37}$, $16{ }^{60} \mathrm{SiQDs}$ were liberated upon alcoholic HF etching for pre-determined times (See: Table S1) and 17 surface functionalized with Si-C tethered 1-dodecyl surface groups via AIBN radical-initiated 18 hydrosilylation. Subsequently, the $\mathrm{d}_{\mathrm{TEM}}$, $\mathrm{d}_{\mathrm{XRD}}$, as well as PL properties were evaluated/compared.

19 This method yielded SiQDs with varying disordered layer thicknesses, where in all cases the $\mathrm{d}_{\text {TEM }}$ 20 is larger than $\mathrm{dXRD}_{\mathrm{X} D}$ (Figure S6). When examining the lifetimes of all of the SiQDs here studied as

21 a function of PL energy, an obvious trend was observed where $\frac{1}{\tau_{S E}}=A * e^{E / E_{0}}$ (Figure S7). This

22 trend is consistent across literature, ${ }^{68,69}$ is independent of synthetic method and has been 23 implicated with the indirect band gap of silicon. ${ }^{70}$ 

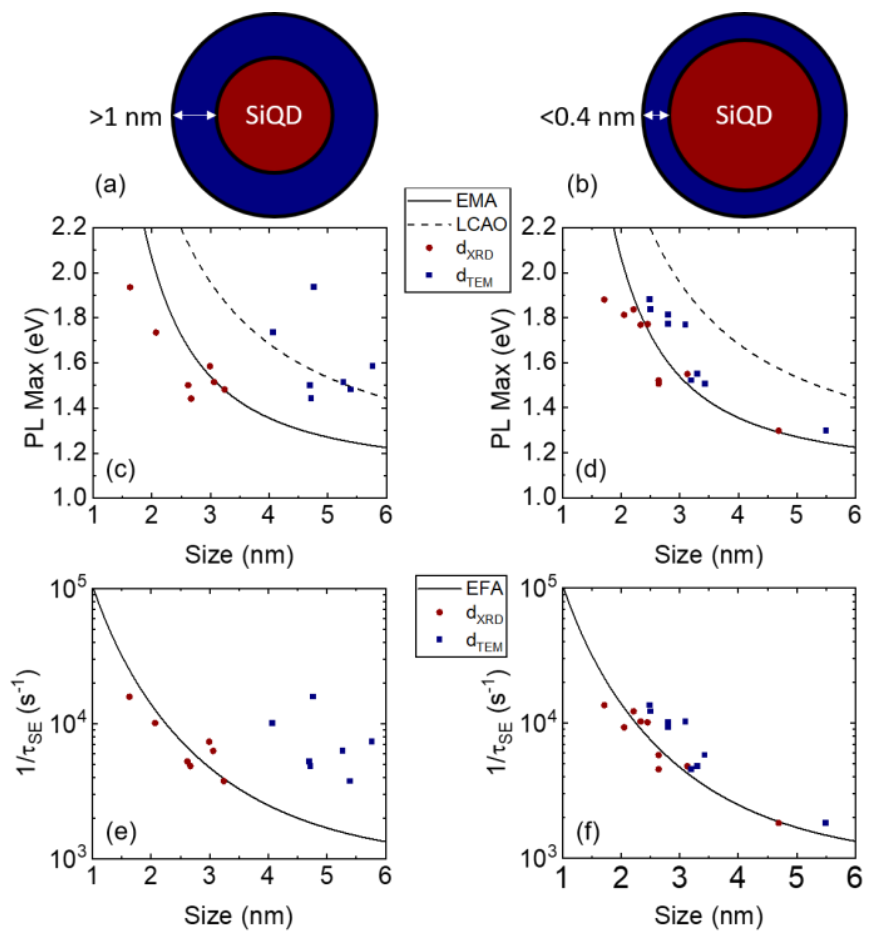

2 Figure 4: A comparison of the relationship between PL energy (c and d) and lifetimes (e and f)

3 with $\mathrm{d}_{\mathrm{TEM}}$ (blue squares) and $\mathrm{d}_{\mathrm{XRD}}$ (red circles) for SiQDs with a thick amorphous layer (>1 nm;

4 a, c, e) and a thin amorphous layer $(<0.4 \mathrm{~nm}$; b, d, f). The solid and dashed black lines in c) and

d) represent the EMA and LCAO as in Figure 1. The solid black line in e) and f) is a fitting from

6 all of the $\mathrm{d}_{\mathrm{XRD}}$ vs. lifetime data according to $\left(\frac{1}{\tau_{S E}}=A\left(\frac{1}{d_{X R D}}\right)^{3}+C\right)$, with $A$ and $C$ being fit

7 parameters.

8 To better illustrate/understand the relationship between SiQD dimensions and optical properties,

9 we categorized the present data into two groupings. 1. Samples in which the d

10 larger (i.e., $\mathrm{d}_{\text {TEM }}-\mathrm{d}_{\mathrm{XRD}}>2 \mathrm{~nm}$ ) than $\mathrm{d}_{\mathrm{XRD}}$. (These particles possess comparatively thick

11 amorphous/disordered Si layers on their surfaces.) 2. Samples for which $\mathrm{d}_{\mathrm{TEM}}$ and $\mathrm{d}_{\mathrm{XRD}}$ are similar

12 (i.e., $\mathrm{d}_{\mathrm{TEM}}-\mathrm{d}_{\mathrm{XRD}}<0.8 \mathrm{~nm}$ ). (These particles possess a thin amorphous/disordered Si layer on their 
1 surfaces.) Data were subsequently plotted and compared with the predictions of the effective mass

2 approximation (EMA; Figures $4 \mathrm{a}$ and $\mathrm{b}$ ).

3 Consistent with our previous observations (see above), there is a clear correlation between $\mathrm{d}_{\mathrm{XRD}}$

4 and EMA predictions for the optical properties of SiQDs in Group 1 (i.e., $\mathrm{d}_{\mathrm{TEM}}-\mathrm{d}_{\mathrm{XRD}}>2 \mathrm{~nm}$;

5 Figure 4a) - this is not the case for $\mathrm{d}_{\text {TEM. }}$ We also note that the EMA consistently overestimates

6 particle size when comparing to $\mathrm{d}_{\mathrm{XRD}}$. In contrast, for Group 2 SiQDs that bear a thin amorphous

7 Si layer, the PL emission energy is closely related to $d_{X R D}$ and $d_{\text {TEM }}$ (Figure $4 b$ ); furthermore, the

8 EMA becomes more representative. We also note similar relationships when evaluating excited-

9 state lifetimes (i.e., $\tau$ ) in the context of $d_{\text {TEM }}$ and $d_{X R D}$.

10 Models have shown that the radiative lifetimes of silicon nanocrystals scale very nearly as the

11 inverse cube of their radius. ${ }^{71,} 72$ While there is also a non-radiative contribution whose

12 contribution cannot be unambiguously determined, the dominant non-radiative decay rate has been

13 calculated to also scale inversely with the crystallite volume. ${ }^{73}$ Thus, the lifetime data was fit to an

14 inverse cubic function $\frac{1}{\tau_{S E}}=A\left(\frac{1}{d_{X R D}}\right)^{3}+C$ of the XRD radius, which appeared to yield a

15 reasonably good fit to the experimental data (Fig. 4(c,d)).

16 The direct relationship between theoretical PL energy and lifetimes and the crystallite diameter of

17 the studied SiQDs, especially when they have a thin amorphous layer (Group 2), suggests that the

18 crystalline core controls the optical response. However, the relationships between SiQD

19 luminescence, graded structure, and size presented for Group 1 are similar to those previously

20 noted for $\mathrm{CdSe} @ \mathrm{CdSe}_{\mathrm{x}} \mathrm{S}_{1-\mathrm{X}} @ \mathrm{CdS} \mathrm{QDs}$ in which the confinement of carriers (i.e., electrons and

21 holes) depends upon a radially varied structure. ${ }^{74}$ A detailed study of the interplay of these factors

22 in the SiQD optical behavior is obviously complicated by the poorly-defined nature of the 
1 transitional region between the crystalline core and amorphous shell, however, one can expect that

2 the confinement should be stronger when the shell is thinner as implied by the results in Fig. 4.

3 This clearly highlights the importance (and promise) of establishing methods for controlling the

4 uniformity of the internal SiQD structure, as well as preparing well-defined core@ shell SiQD

5 systems.

\section{Conclusions}

7 In conclusion, the optical properties of functionalized SiQDs have been evaluated in the context

8 of internal crystallinity by examining crystallite and particle dimensions using X-ray diffraction

9 and transmission electron microscopy. For SiQDs prepared via the widely employed "HSQ

10 method", the $\mathrm{d}_{\mathrm{XRD}}$ provides a better representation of the optically active QD dimensions,

11 regardless of the presence of an amorphous overlayer. In cases in which a thick $\mathrm{Si}$ amorphous

12 layer is present, no obvious correlation between $\mathrm{d}_{\mathrm{TEM}}$ and optical response is noted. However,

13 EMA estimates derived from $\mathrm{d}_{\mathrm{TEM}}$ and $\mathrm{d}_{\mathrm{XRD}}$ dimensions agree when thin amorphous Si layers are

14 present. This fundamental understanding of SiQD structure and its influence on their optical 15 properties illuminates a foundation on which future efforts to better control SiQD optical response 16 can build.

\section{Experimental}

18 Materials:

19 Reagents:

20 Hydrofluoric acid (Electronic grade, 48-50\%) was purchased from Fischer Scientific. Sulfuric 21 acid (reagent grade, 95-98\%) was purchased from Caledon Laboratory Chemicals. Fuming 22 sulfuric acid (reagent grade, $20 \%$ free $\mathrm{SO}_{3}$ bases) and trichlorosilane (99\%) were purchased from 23 Sigma (now MilliporeSigma). All reagents were used as received unless otherwise specified. All 
1 solvents were reagent grade and used as received. Toluene was collected from a Pure-Solv

2 purification system immediately prior to use. Benzene was purchased from EMD Millipore (now

3 Millipore Sigma).

$4 \quad$ Preparation of Hydrogen Silsesquioxane (HSQ):

5 HSQ was synthesized via known literature procedures where sulfuric acid is used to selectively

6 oxidize trichlorosilane. Dry toluene $(45.0 \mathrm{~mL})$ was added to a mixture of concentrated $(15.0 \mathrm{~mL})$

7 and fuming $(7.2 \mathrm{~mL})$ sulfuric acid under inert atmosphere. Once the addition was complete, 110

$8 \mathrm{~mL}$ of dry toluene was added to $16 \mathrm{~mL}$ of trichlorosilane and added drop-wise over a few hours to

9 the sulfuric acid solution. The product dissolved in the organic layer was washed with sulfuric acid

10 solution. After drying the organic layer, most of the toluene was removed via rotary evaporation

11 and the rest was removed in vacuo. The resulting white solid was stored under vacuum until use.

12 Preparation of the $\mathrm{SiQDs} / \mathrm{SiO}_{2}$ composite:

13 Thermal decomposition of the HSQ, as previously reported, was used to produce the SiQDs used

14 in this study. Six grams of HSQ was annealed in a tube furnace under a $5 \% \mathrm{H}_{2} / \mathrm{Ar}$ atmosphere at

151100,1200 , and $1300{ }^{\circ} \mathrm{C}$ to get various sizes of nanocrystals (the sizes associated with each

16 temperature can be seen in Table S1). The composite was ground using an agate mortar and pestle

17 then shaken for six hours to prepare a fine powder.

18 Preparation of alkyl passivated SiQDs:

19 The composite was then etched using a 1:1:1 solution of ethanol:DI water:HF to remove the $\mathrm{SiO}_{2}$

20 matrix from the particles using $0.5 \mathrm{~g}$ of composite per etch and $15 \mathrm{~mL}$ total etching solution. The

$21 \quad 1100{ }^{\circ} \mathrm{C}$ composite was etched for one hour (one hour and forty-five minutes to two hours and

22 forty-five minutes for $1200{ }^{\circ} \mathrm{C}$ composite) and the resulting particles were extracted in toluene.

23 The hydride-terminates SiQDs (H-SiQDs) were centrifuged twice in toluene and redispersed in 10 
$1 \mathrm{~mL}$ dry toluene with $6 \mathrm{~mL}$ of dodecene and $300 \mathrm{mg}$ AIBN. The reaction mixture was degassed

2 using three freeze-pump-thaw cycles and placed in an oil bath at $70{ }^{\circ} \mathrm{C}$ overnight ( $\sim 17$ hours).

3 The resulting SiQDs were purified via centrifugation using $10 \mathrm{~mL}$ of toluene and $20 \mathrm{~mL}$ of

4 methanol thrice to remove any unreacted dodecene and AIBN. The purified SiQDs were

5 redispersed in toluene for subsequent characterization.

\section{Characterization:}

$7 \quad \underline{\text { FTIR }}$

8 Fourier transform infrared spectroscopy (FTIR) was performed on a Thermo Nicolet Continum

$9 \quad$ FT-IR microscope by drop casting SiQDs onto a silicon wafer from dry toluene solutions.

$10 \underline{X P S}$

11 X-ray photoelectron spectroscopy (XPS) was measured using a Kratos Axis 165 Ultra X-ray

12 photoelectron spectrometer. A monochromatic $\mathrm{Al} \mathrm{K \alpha}$ source operating at $210 \mathrm{~W}$ with an energy

$13 \mathrm{~h} v=1486.6 \mathrm{eV}$ was used. Survey spectra were collected with an analyzer pass energy of $160 \mathrm{eV}$

14 and step of $0.3 \mathrm{eV}$. For high-resolution spectra, the pass energy was $20 \mathrm{eV}$ and the step was 0.1

$15 \mathrm{eV}$ with dwell time of $200 \mathrm{~ms}$. XPS samples were prepared by drop-coating a dispersion of SiQDs

16 in dry toluene onto a copper foil. Spectra were calibrated to the $\mathrm{C} 1 \mathrm{~s}(284.8 \mathrm{eV})$ and fit to

17 appropriate spin-orbit pairs using CasaXPS (VAMAS) software with a Shirley-type background.

18 To fit the Si $2 p$ high resolution spectrum the doublet area ratio was fixed at 2:1 and the separation

19 was set at 0.6 .

20 Electron Microscopy

21 Transmission electron microscopy (TEM) and HR-TEM were performed on a JEOL JEM-

22 ARM200CF S/TEM (Cold Field Emission Gun) electron microscope with an accelerating voltage

23 of $200 \mathrm{kV}$ using SiQDs drop-coated from a toluene solution onto a holey carbon-coated copper 
1 grid. The SiQD size was determined by averaging the size of 300 particles using ImageJ software

2 (version $1.51 \mathrm{j} 8)$.

$3 \underline{\mathrm{XRD}}$

4 Powder X-ray diffraction was measured on a Rigaku Ultima IV multipurpose X-ray diffraction

5 system using a $\mathrm{Cu} \mathrm{K \alpha}$ source for most of the samples. The sample was drop cast on a zero-

6 background Si wafer and collected as a thin film. For thin film diffraction, a parallel beam as used

7 with a glancing angle of $0.5^{\circ}$. Some of the data was also collected at the synchrotron, with a

8 wavelength of $0.6892 \AA$, using transmission mode, to verify the size. These methods gave the same

9 results within the error of the technique and the fitting. This is likely because the line width due to

10 size broadening is significantly larger than the instrument broadening for either of these methods.

11 Pawley fitting of powder pattern was performed on TOPAS was used to determine the crystallite

12 domain size with integral breadth, FWHM and Lorentzian broadening methods. Instrumental

13 effects were accounted for by refining a NIST Si line shape standard (640f) to ensure instrument

14 alignment and line shape. A sample-independent synthetic peak was required at $\sim 22^{\circ}$ for all

15 measurements on the laboratory instrument, which is believed to originate from the sample holder

16 imperfections or amorphous products/unreacted materials. The synchrotron data required other

17 small peaks $\left(8,9,11,14,16\right.$, and $\left.19^{\circ}\right)$ due to imperfections in the background subtraction from the

18 Kapton tube (sample holder).

19 Photoluminescence characterization

20 Photoluminescence spectroscopy measurements were performed on a solution of silicon

21 nanocrystals dispersed in toluene in a quartz cuvette. Silicon nanoparticles were excited using an

22 argon ion laser with a $351 \mathrm{~nm}$ emission wavelength. The resulting photoluminescence was

23 collected by an optic fiber, passed through a $500 \mathrm{~nm}$ long-pass filter to eliminate scattered light 
1 from the excitation source, and fed into an Ocean Optics USB2000 spectrometer. The spectral

2 response was calibrated by a blackbody radiator (Ocean Optics LS1). Photoluminescence lifetime

3 measurements were acquired using an $\operatorname{argon}$ ion laser $(351 \mathrm{~nm}, \sim 20 \mathrm{~mW})$ modulated by an acousto-

4 optic modulator ( $\sim 50 \mathrm{~ns}$ response time) operated at a frequency of $200 \mathrm{~Hz}$ with a $50 \%$ duty cycle.

5 The photoluminescence was fed into an optic fiber and passed through a $500 \mathrm{~nm}$ long-pass filter

6 and was then incident on a Hamamatsu H7422P-50 photomultiplier tube (PMT) interfaced with a

7 Becker-Hickl PMS-400A gated photon counter. The photoluminescence data was collected with a

8 total of 10000 sweeps for good signal-to-noise ratio and using $1 \mu$ s time steps. A log-normal fitting

9 of the data in MatLab was used to calculate the luminescence decay.

10 Quantum efficiency measurements were performed using a homemade integrating sphere with a

$11365 \mathrm{~nm}$ light emitting diode excitation source. Sample solutions were diluted to have an

12 absorbance between 0.1 and 0.15 at $405 \mathrm{~nm}$, then they were transferred into a cuvette that was

13 lowered into the integrating sphere on a magnetic holder as was a distilled water blank. The

14 photoluminescence and excitation intensities were captured through a fiber attached to the sphere

15 and analyzed with a calibrated Ocean Optics spectrometer. The equation QE =

$16\left(I_{\mathrm{PL}, \text { sample }}-I_{\mathrm{PL}, \text { blank }}\right) /\left(I_{\mathrm{ex}, \text { blank }}-I_{\mathrm{ex}, \text { sample }}\right)$ was used to calculate the absolute quantum efficiency, were

$17 I_{\mathrm{PL}}$ is integrated photoluminescence intensity and $I_{\mathrm{ex}}$ is excitation intensity recorded inside the

18 sphere. The measurements were performed in quintuplicates.

19 Acknowledgments

20 The Natural Science and Engineering Research Council (NSERC) of Canada Discovery Grants

21 Program (RGPIN-2015-03896) and the University of Alberta are acknowledged for their generous

22 research support. A. Thiessen is supported by the Alberta Innovates Graduate Student Scholarship.

23 L. Zhang is supported by China Scholarship Council (2018-31010). The ATUMS training program 
1 is supported by NSERC CREATE and is thanked for continued generous financial support

2 (CREATE-463990-2015). Part of the research described in this paper was performed at the

3 Canadian Light Source, a national research facility of the University of Saskatchewan, which is

$4 \quad$ supported by the Canada Foundation for Innovation (CFI), the Natural Sciences and Engineering

5 Research Council (NSERC), the National Research Council (NRC), the Canadian Institutes of

6 Health Research (CIHR), the Government of Saskatchewan, and the University of Saskatchewan.

7 References

8 1. Samsung Newsroom. [NEXT for QLED] Part 1: Samsung Dominates the Large Screen TV 9 Market. https://news.samsung.com/global/next-for-qled-part-1-samsung-dominates-the-large10 screen-tv-market (01/02),

$112 . \quad$ Halford, B., Quantum dot LEDs go cadmium-free. In American Chemical Society: Chemical 12 and Engineering News, 2019; Vol. 97.

13 3. Union, T. E., Ed. Publications Office of the European Union: Official Journal of the European 14 Union, 2017; Vol. 2017/1975, p 3.

4. Program, N. T. NTP Technical Report on the Toxicology and Carcinogenesis Studies of Indium Phosphide in F344/N Rats and B6C3F1 Mice (Inhalation Studies); U.S. Department of Health and Human Services: July 2001, 2001.

5. Nanoco https://www.nanocotechnologies.com/ (01/02),

6. Veinot, J. G. C., Synthesis, surface functionalization, and properties of freestanding silicon nanocrystals. Chem. Commun. 2006, 40, 4160-4168.

7. Mangolini, L.; Kortshagen, U., Plasma-assisted synthesis of silicon nanocrystal inks. Adv. Mater. 2007, 19 (18), 2513-2519.

23 8. Tilley, R. D.; Warner, J. H.; Yamamoto, K.; Matsui, I.; Fujimori, H., Micro-emulsion 24 synthesis of monodisperse surface stabilized silicon nanocrystals. Chem. Commun. 2005, 14, $25 \quad 1833-1835$.

26 9. Baldwin, R. K.; Pettigrew, K. A.; Ratai, E.; Augustine, M. P.; Kauzlarich, S. M., Solution 27 reduction synthesis of surface stabilized silicon nanoparticles. Chem. Commun. 2002, 17, 18221823.

10. Zou, J.; Kauzlarich, S. M., Functionalization of Silicon Nanoparticles via Silanization: Alkyl, Halide and Ester. J. Clust. Sci. 2008, 19 (2), 341-355.

11. Yang, Z.; Gonzalez, C. M.; Purkait, T. K.; Iqbal, M.; Meldrum, A.; Veinot, J. G., Radical Initiated Hydrosilylation on Silicon Nanocrystal Surfaces: An Evaluation of Functional Group Tolerance and Mechanistic Study. Langmuir 2015, 31 (38), 10540-10548. 12. Liu, X.; Zhao, S.; Gu, W.; Zhang, Y.; Qiao, X.; Ni, Z.; Pi, X.; Yang, D., Light-Emitting Diodes Based on Colloidal Silicon Quantum Dots with Octyl and Phenylpropyl Ligands. ACS Appl. Mater. Interfaces 2018, 10 (6), 5959-5966. 
13. Meinardi, F.; Ehrenberg, S.; Dhamo, L.; Carulli, F.; Mauri, M.; Bruni, F.; Simonutti, R.; Kortshagen, U.; Brovelli, S., Highly efficient luminescent solar concentrators based on earthabundant indirect-bandgap silicon quantum dots. Nature Photon. 2017, 11, 177-185.

14. Mazzaro, R.; Gradone, A.; Angeloni, S.; Morselli, G.; Cozzi, P. G.; Romano, F.; Vomiero, A.; Ceroni, P., Hybrid Silicon Nanocrystals for Color-Neutral and Transparent Luminescent Solar Concentrators. ACS Photonics 2019, 6 (9), 2303-2311.

15. Erogbogbo, F.; Yong, K.-T.; Roy, I.; Hu, R.; Law, W.-C.; Zhao, W.; Ding, H.; Wu, F.; Kumar, R.; Swihart, M. T.; Prasad, P. N., In Vivo Targeted Cancer Imaging, Sentinel Lymph Node Mapping and Multi-Channel Imaging with Biocompatible Silicon Nanocrystals. ACS Nano 2011, 5 (1), 413423.

16. Tu, C.; Ma, X.; House, A.; Kauzlarich, S. M.; Louie, A. Y., PET Imaging and Biodistribution of Silicon Quantum Dots in Mice. ACS Med. Chem. Lett. 2011, 2 (4), 285-288.

17. Gonzalez, C. M.; Iqbal, M.; Dasog, M.; Piercey, D. G.; Lockwood, R.; Klapotke, T. M.; Veinot, J. G. C., Detection of high-energy compounds using photoluminescent silicon nanocrystal paper based sensors. Nanoscale 2014, 6 (5), 2608-2612.

18. Gonzalez, C.M.; Veinot, J. G. C., Silicon nanocrystals for the development of sensing platforms. J. Mater. Chem. C 2016, 4 (22), 4836-4846.

19. Kim, H.; Seo, M.; Park, M. H.; Cho, J., A critical size of silicon nano-anodes for lithium rechargeable batteries. Angew. Chem. Int. Ed. 2010, 49 (12), 2146-2149.

20. Su, X.; Wu, Q.; Li, J.; Xiao, X.; Lott, A.; Lu, W.; Sheldon Brian, W.; Wu, J., Silicon-Based Nanomaterials for Lithium-Ion Batteries: A Review. Adv. Energy Mater. 2014, 4 (1), 1300882.

21. Dasog, M.; De los Reyes, G. B.; Titova, L. V.; Hegmann, F. A.; Veinot, J. G. C., Size vs surface: tuning the photoluminescence of freestanding silicon nanocrystals across the visible spectrum via surface groups. ACS Nano 2014, 8 (9), 9636-48.

22. Mobarok, M. H.; Purkait, T. K.; Islam, M. A.; Miskolzie, M.; Veinot, J. G. C., Instantaneous Functionalization of Chemically Etched Silicon Nanocrystal Surfaces. Angew. Chem. Int. Ed. 2017, 56 (22), 6073-6077.

23. Islam, M. A.; Mobarok, M. H.; Sinelnikov, R.; Purkait, T. K.; Veinot, J. G. C., Phosphorus Pentachloride Initiated Functionalization of Silicon Nanocrystals. Langmuir 2017, 33 (35), 87668773.

24. Sangghaleh, F.; Sychugov, I.; Yang, Z.; Veinot, J. G. C.; Linnros, J., Near-Unity Internal Quantum Efficiency of Luminescent Silicon Nanocrystals with Ligand Passivation. ACS Nano 2015, 9 (7), 7097-7104.

25. Jurbergs, D.; Rogojina, E.; Mangolini, L.; Kortshagen, U., Silicon nanocrystals with ensemble quantum yields exceeding 60\%. Appl. Phys. Lett. 2006, 88 (23), 233116.

26. Wheeler, L. M.; Anderson, N. C.; Palomaki, P. K. B.; Blackburn, J. L.; Johnson, J. C.; Neale, N. R., Silyl Radical Abstraction in the Functionalization of Plasma-Synthesized Silicon Nanocrystals. Chem. Mater. 2015, 27 (19), 6869-6878.

27. Ddungu, J. L. Z.; Silvestrini, S.; Tassoni, A.; De Cola, L., Shedding Light on the Aqueous Synthesis of Silicon Nanoparticles by Reduction of Silanes with Citrates. Faraday Discussions 2020. Accepted Manuscript DOI:10.1039/C9FD00127A

28. Dasog, M.; Yang, Z. Y.; Regli, S.; Atkins, T. M.; Faramus, A.; Singh, M. P.; Muthuswamy, E.; Kauzlarich, S. M.; Tilley, R. D.; Veinot, J. G. C., Chemical Insight into the Origin of Red and Blue 
Photoluminescence Arising from Freestanding Silicon Nanocrystals. ACS Nano 2013, 7 (3), 26762685.

29. Sinelnikov, R.; Dasog, M.; Beamish, J.; Meldrum, A.; Veinot, J. G. C., Revisiting an Ongoing Debate: What Role Do Surface Groups Play in Silicon Nanocrystal Photoluminescence? ACS Photonics 2017, 4 (8), 1920-1929.

30. Rodríguez, J. A.; Vásquez-Agustín, M. A.; Morales-Sánchez, A.; Aceves-Mijares, M., Emission Mechanisms of Si Nanocrystals and Defects in $\mathrm{SiO}_{2}$ Materials. J. Nanomater. 2014, 2014, 409482.

31. Canham, L. T., Luminescence Bands and their Proposed Origins In Highly Porous Silicon. phys. stat. sol. (b) 1995, 190, 9-14.

32. Lee, B. G.; Luo, J.-W.; Neale, N. R.; Beard, M. C.; Hiller, D.; Zacharias, M.; Stradins, P.; Zunger, A., Quasi-Direct Optical Transitions in Silicon Nanocrystals with Intensity Exceeding the Bulk. Nano Lett. 2016, 16 (3), 1583-1589.

33. Mazzaro, R.; Romano, F.; Ceroni, P., Long-lived Luminescence of Silicon Nanocrystals: From Principles to Applications. Phys. Chem. Chem. Phys. 2017, 19 (39), 26507-26526.

34. DeBenedetti, W. J. I.; Chiu, S.-K.; Radlinger, C. M.; Ellison, R. J.; Manhat, B. A.; Zhang, J. Z.; Shi, J.; Goforth, A. M., Conversion from Red to Blue Photoluminescence in Alcohol Dispersions of Alkyl-Capped Silicon Nanoparticles: Insight into the Origins of Visible Photoluminescence in Colloidal Nanocrystalline Silicon. J. Phys. Chem. C 2015, 119 (17), 9595-9608.

35. Dasog, M.; Bader, K.; Veinot, J. G. C., Influence of Halides on the Optical Properties of Silicon Quantum Dots. Chem. Mater. 2015, 27 (4), 1153-1156.

36. Li, X.; He, Y.; Talukdar, S. S.; Swihart, M. T., Process for Preparing Macroscopic Quantities of Brightly Photoluminescent Silicon Nanoparticles with Emission Spanning the Visible Spectrum. Langmuir 2003, 19 (20), 8490-8496.

37. Hessel, C. M.; Reid, D.; Panthani, M. G.; Rasch, M. R.; Goodfellow, B. W.; Wei, J.; Fujii, H.; Akhavan, V.; Korgel, B. A., Synthesis of Ligand-Stabilized Silicon Nanocrystals with SizeDependent Photoluminescence Spanning Visible to Near-Infrared Wavelengths. Chem. Mater. 2012, 24 (2), 393-401.

38. Niquet, Y. M.; Delerue, C.; Allan, G.; Lannoo, M., Method for Tight-binding Parametrization: Application to Silicon Nanostructures. Phys. Rev. B 2000, 62 (8), 5109-5116.

39. Yu, Y.; Fan, G.; Fermi, A.; Mazzaro, R.; Morandi, V.; Ceroni, P.; Smilgies, D.-M.; Korgel, B. A., Size-Dependent Photoluminescence Efficiency of Silicon Nanocrystal Quantum Dots. J. Phys. Chem. C 2017, 121 (41), 23240-23248.

40. Jia, X.; Zhang, P.; Lin, Z.; Anthony, R.; Kortshagen, U.; Huang, S.; Puthen-Veettil, B.; Conibeer, G.; Perez-Wurfl, I., Accurate Determination of the Size Distribution of Si Nanocrystals from PL Spectra. RSC Adv. 2015, 5 (68), 55119-55125.

41. de Boer, W. D. A. M.; Timmerman, D.; Dohnalová, K.; Yassievich, I. N.; Zhang, H.; Buma, W. J.; Gregorkiewicz, T., Red Spectral Shift and Enhanced Quantum Efficiency in Phonon-Free Photoluminescence from Silicon Nanocrystals. Nat. Nanotechnol. 2010, 5 (12), 878-884.

42. Delerue, C.; Allan, G.; Lannoo, M., Theoretical Aspects of the Luminescence of Porous Silicon. Phys. Rev. B 1993, 48 (15), 11024-11036.

43. Wang, L. W.; Zunger, A., Electronic Structure Pseudopotential Calculations of Large (.apprx.1000 Atoms) Si Quantum Dots. J. Phys. Chem. 1994, 98 (8), 2158-2165. 
44. Hill, N. A.; Whaley, K. B., Size Dependence of Excitons in Silicon Nanocrystals. Phys. Rev. Lett. 1995, 75 (6), 1130-1133.

45. Hill, N. A.; Whaley, K. B., A Theoretical Study of Light Emission from Nanoscale Silicon. J. Electron. Mater. 1996, 25 (2), 269-285.

46. Öğüt, S.; Chelikowsky, J. R.; Louie, S. G., Quantum Confinement and Optical Gaps in Si Nanocrystals. Phys. Rev. Lett. 1997, 79 (9), 1770-1773.

47. Liu, S.-M.; Yang; Sato, S.; Kimura, K., Enhanced Photoluminescence from Si Nanoorganosols by Functionalization with Alkenes and Their Size Evolution. Chem. Mater. 2006, 18 (3), 637-642.

48. Mastronardi, M. L.; Maier-Flaig, F.; Faulkner, D.; Henderson, E. J.; Kübel, C.; Lemmer, U.; Ozin, G. A., Size-Dependent Absolute Quantum Yields for Size-Separated Colloidally-Stable Silicon Nanocrystals. Nano Lett. 2012, 12 (1), 337-342.

49. Hannah, D. C.; Yang, J.; Podsiadlo, P.; Chan, M. K. Y.; Demortière, A.; Gosztola, D. J.; Prakapenka, V. B.; Schatz, G. C.; Kortshagen, U.; Schaller, R. D., On the Origin of Photoluminescence in Silicon Nanocrystals: Pressure-Dependent Structural and Optical Studies. Nano Lett. 2012, 12 (8), 4200-4205.

50. Wen, X.; Zhang, P.; Smith, T. A.; Anthony, R. J.; Kortshagen, U. R.; Yu, P.; Feng, Y.; Shrestha, S.; Coniber, G.; Huang, S., Tunability Limit of Photoluminescence in Colloidal Silicon Nanocrystals. Sci. Rep. 2015, 5 (1), 12469.

51. Jakob, M.; Javadi, M.; Veinot, J. G. C.; Meldrum, A.; Kartouzian, A.; Heiz, U., Ensemble Effects in the Temperature-Dependent Photoluminescence of Silicon Nanocrystals. Chem. Eur. J. 2019, 25 (12), 3061-3067.

52. Breitenecker, M.; Sexl, R.; Thirring, W., On the effective mass approximation. Z. Physik 1964, 182 (2), 123-129.

53. Hannah, D. C.; Yang, J.; Kramer, N. J.; Schatz, G. C.; Kortshagen, U. R.; Schaller, R. D., Ultrafast Photoluminescence in Quantum-Confined Silicon Nanocrystals Arises from an Amorphous Surface Layer. ACS Photonics 2014, 1, (10), 960-967.

54. Kůsová, K.; Ondič, L.; Pelant, I., Comment on "Ultrafast Photoluminescence in QuantumConfined Silicon Nanocrystals Arises from an Amorphous Surface Layer". ACS Photonics 2015, 2 (3), 454-455.

55. Hannah, D. C.; Yang, J.; Kramer, N. J.; Schatz, G. C.; Kortshagen, U. R.; Schaller, R. D., Reply to "Comment on 'Ultrafast Photoluminescence in Quantum-Confined Silicon Nanocrystals Arises from an Amorphous Surface Layer'”. ACS Photonics 2015, 2 (3), 456-458.

34

56. Brown, S. L.; Miller, J. B.; Anthony, R. J.; Kortshagen, U. R.; Kryjevski, A.; Hobbie, E. K., Abrupt Size Partitioning of Multimodal Photoluminescence Relaxation in Monodisperse Silicon Nanocrystals. ACS Nano 2017, 11 (2), 1597-1603.

57. Thiessen, A. N.; Ha, M.; Hooper, R. W.; Yu, H.; Oliynyk, A. O.; Veinot, J. G. C.; Michaelis, V. K., Silicon Nanoparticles: Are They Crystalline from the Core to the Surface? Chem. Mater. 2019, 31 (3), 678-688.

40 58. Jarolimek, K.; Hazrati, E.; de Groot, R. A.; de Wijs, G. A., Band Offsets at the Interface between Crystalline and Amorphous Silicon from First Principles. Phys. Rev. Applied 2017, 8 (1), 014026. 
59. Hessel, C. M.; Henderson, E. J.; Veinot, J. G. C., Hydrogen Silsesquioxane: A Molecular Precursor for Nanocrystalline Si-SiO2 Composites and Freestanding Hydride-Surface-Terminated Silicon Nanoparticles. Chem. Mater. 2006, 18 (26), 6139-6146.

60. Hessel, C. M.; Henderson, E. J.; Veinot, J. G. C., An Investigation of the Formation and Growth of Oxide-Embedded Silicon Nanocrystals in Hydrogen Silsesquioxane-Derived Nanocomposites. J. Phys. Chem. C 2007, 111 (19), 6956-6961.

61. Clark, R. J.; Aghajamali, M.; Gonzalez, C. M.; Hadidi, L.; Islam, M. A.; Javadi, M.; Mobarok, M. H.; Purkait, T. K.; Robidillo, C. J. T.; Sinelnikov, R.; Thiessen, A. N.; Washington, J.; Yu, H.; Veinot, J. G. C., From Hydrogen Silsesquioxane to Functionalized Silicon Nanocrystals. Chem. Mater. 2017, 29 (1), 80-89.

62. Dohnalova, K.; Gregorkiewicz, T.; Kusova, K., Silicon quantum dots: surface matters. J. Phys. Condens. Matter 2014, 26 (17), 173201.

63. Balzar, D.; Audebrand, N.; Daymond, M. R.; Fitch, A.; Hewat, A.; Langford, J. I.; Le Bail, A.; Louër, D.; Masson, O.; McCowan, C. N.; Popa, N. C.; Stephens, P. W.; Toby, B. H., Size-strain linebroadening analysis of the ceria round-robin sample. J. Appl. Cryst. 2004, 37 (6), 911-924.

64. Jakob, M.; Aissiou, A.; Morrish, W.; Marsiglio, F.; Islam, M.; Kartouzian, A.; Meldrum, A., Reappraising the Luminescence Lifetime Distributions in Silicon Nanocrystals. Nanoscale Res. Lett. 2018, 13 (1), 383.

65. Kim, S.; Fisher, B.; Eisler, H.-J.; Bawendi, M., Type-Il Quantum Dots: CdTe/CdSe(Core/Shell) and CdSe/ZnTe(Core/Shell) Heterostructures. J. Am. Chem. Soc. 2003, 125 (38), 11466-11467.

66. Lee, B. G.; Hiller, D.; Luo, J. W.; Semonin, O. E.; Beard, M. C.; Zacharias, M.; Stradins, P., Strained Interface Defects in Silicon Nanocrystals. Adv. Funct. Mater. 2012, 22 (15), 3223-3232.

67. Kleovoulou, K.; Kelires, P. C., Stress state of embedded Si nanocrystals. Phys. Rev. B 2013, $88,085424$.

68. Miller, J. B.; Van Sickle, A. R.; Anthony, R. J.; Kroll, D. M.; Kortshagen, U. R.; Hobbie, E. K., Ensemble Brightening and Enhanced Quantum Yield in Size-Purified Silicon Nanocrystals. ACS Nano 2012, 6 (8), 7389-7396.

69. Huisken, F.; Ledoux, G.; Guillois, O.; Reynaud, C., Light-Emitting Silicon Nanocrystals from Laser Pyrolysis. Adv. Mater. 2002, 14 (24), 1861-1865.

70. Delerue, C.; Allan, G.; Reynaud, C.; Guillois, O.; Ledoux, G.; Huisken, F., Multiexponential Photoluminescence Decay in Indirect-Gap Semiconductor Nanocrystals. Phys. Rev. B 2006, 73 (23), 235318.

71. Belyakov, V. A.; Burdov, V. A.; Gaponova, D. M.; Mikhaylov, A. N.; Tetelbaum, D. I.; Trushin, S. A., Phonon-Assisted Radiative Electron-Hole Recombination in Silicon Quantum Dots. Phys. Solid State 2004, 46, 27-31.

72. Belyakov, V. A.; Burdov, V. A.; Lockwood, R.; Meldrum, A., Silicon Nanocrystals: Fundamental Theory and Implications for Stimulated Emission. Adv. Opt. Technol. 2008, 2008, 279502.

73. Lannoo, M.; Delerue, C.; Allan, G., Nonradiative Recombination on Dangling Bonds in Silicon Crystallites. J. Lumin. 1993, 57 (1), 243-247.

74. Bae, W. K.; Padilha, L. A.; Park, Y.-S.; McDaniel, H.; Robel, I.; Pietryga, J. M.; Klimov, V. I., Controlled Alloying of the Core-Shell Interface in CdSe/CdS Quantum Dots for Suppression of Auger Recombination. ACS Nano 2013, 7 (4), 3411-3419. 
1 
Supplemental Information

\section{A Tale of Seemingly "Identical” Silicon Quantum}

\section{Dot Families: Structural Insight into Silicon}

\section{Quantum Dot Photoluminescence.}

Alyxandra N. Thiessen ${ }^{a}$, Lijuan Zhang ${ }^{b}$, Anton O. Oliynyk ${ }^{c}$, Haoyang Yu ${ }^{a}$, Kevin M. O'Connor ${ }^{a}$, Alkiviathes Meldrum ${ }^{b}$, and Jonathan G. C. Veinot ${ }^{a^{*}}$

${ }^{a}$ Department of Chemistry, University of Alberta, Edmonton, Alberta T6G 2G2, Canada

${ }^{b}$ Department of Physics, University of Alberta, Edmonton, Alberta T6G 2E1, Canada

${ }^{c}$ Manhattan College, Riverdale, New York, United States, 10471

* Corresponding author's email: jveinot@ualberta.ca 


\section{Interpreting luminescence lifetimes:}

To understand the luminescence lifetimes, the decays were fit with equation S1. ${ }^{1}$

$$
I_{t}=A \exp \left[-\left(t / \tau_{D}\right)^{\beta}\right]+\mathrm{dc}
$$

For this function, the mean lifetime is given by equation $\mathrm{S} 2$.

(2)

$$
\tau_{S E}=\tau_{D} \frac{\Gamma(2 / \beta)}{\Gamma(1 / \beta)}
$$

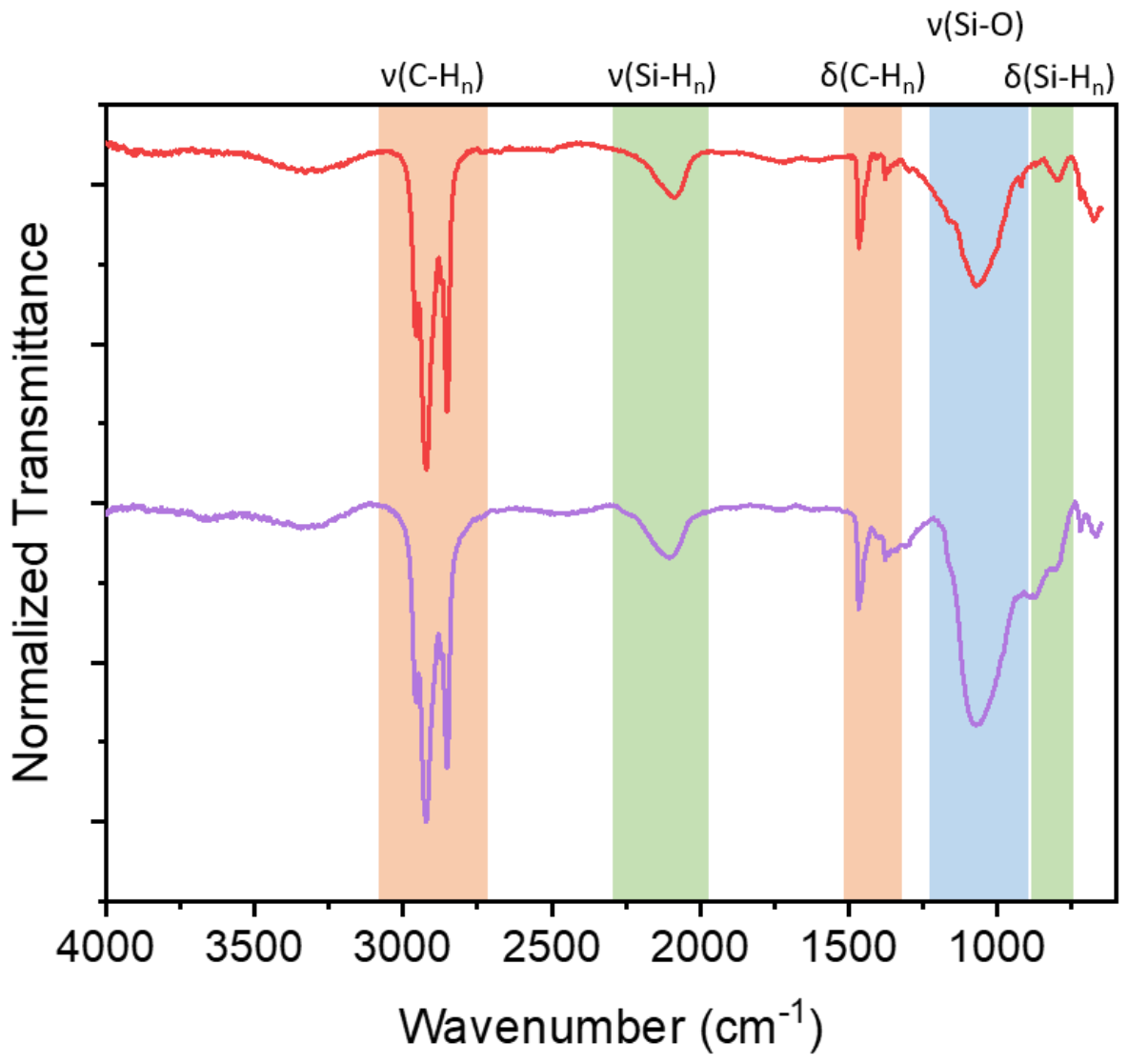

Figure S1: FTIR of 1200-SiQDs (red) and 1300-SiQDs (purple). 

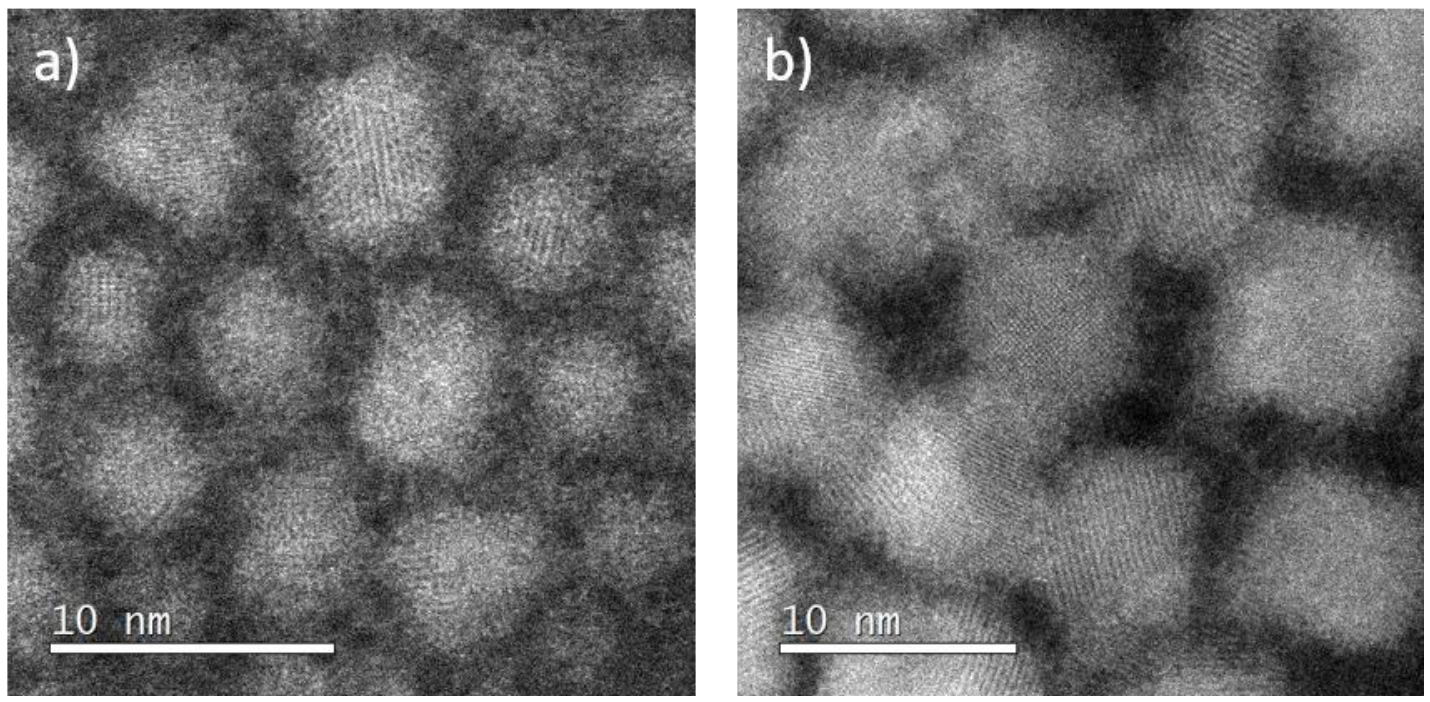

Figure S2: High annular angle dark field STEM images of a) 1200-SiQDs and b) 1300-SiQDs.
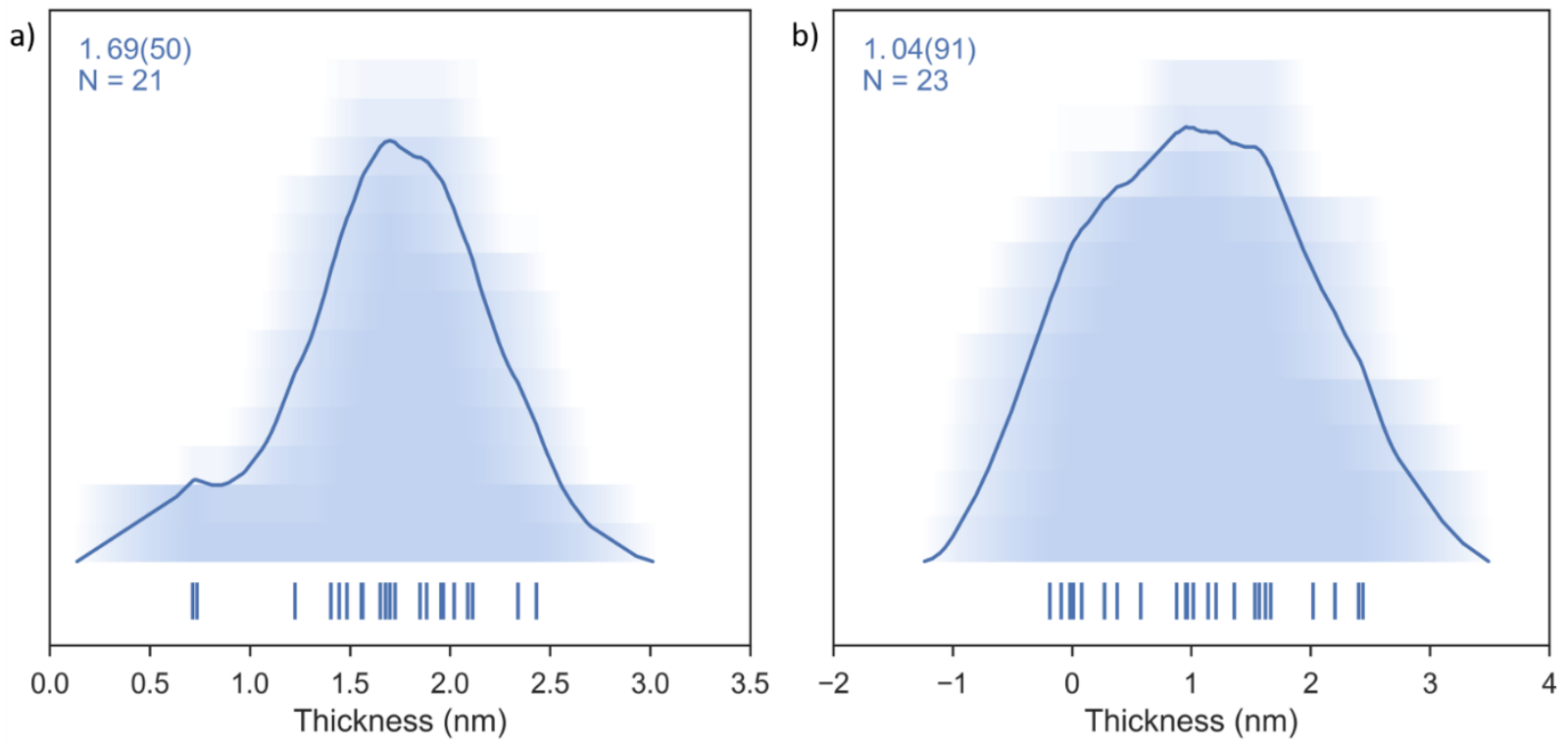

Figure S3: Histograms showing the STEM determined non-crystalline shell thicknesses for a) 1200-SiQDs and b) 1300-SiQDs. The numbers represent the average amorphous shell thickness measured over $\mathrm{N}$ samples. 


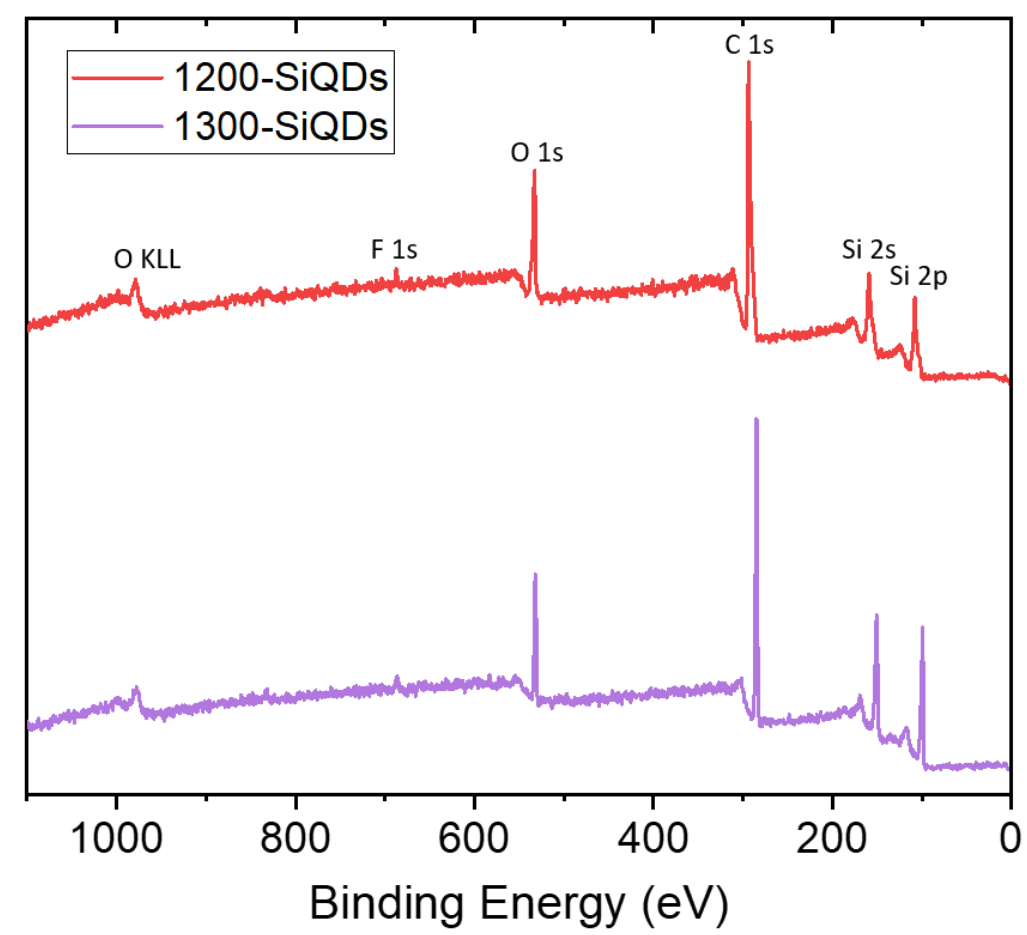

Figure S4: Survey XPS scan for a) 1200-SiQDs and b) 1300-SiQDs, showing the presence of only Si, C, O and $F$.
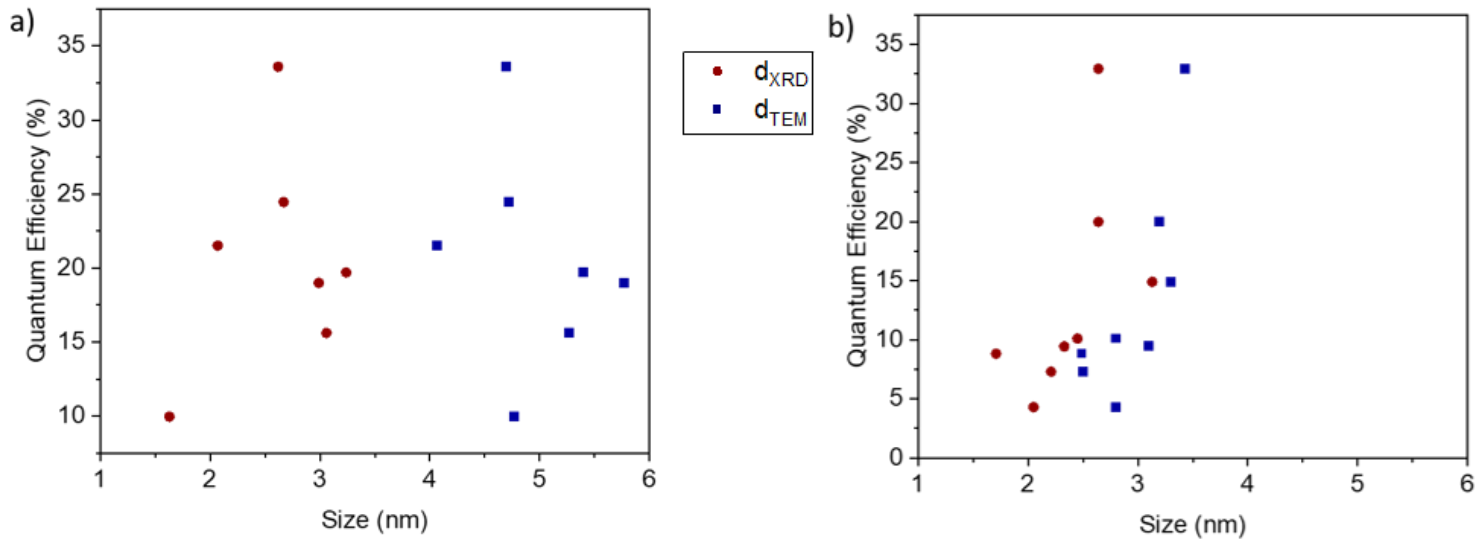

Figure S5: A comparison of the relationship between quantum efficiency with $\mathrm{d}_{\text {TEM }}$ (blue squares) and $\mathrm{d}_{\mathrm{XRD}}$ (red circles) for SiQDs with a thick amorphous layer ( $>2 \mathrm{~nm}$; a) and a thin amorphous layer $(<0.8 \mathrm{~nm} ; \mathrm{b})$. 


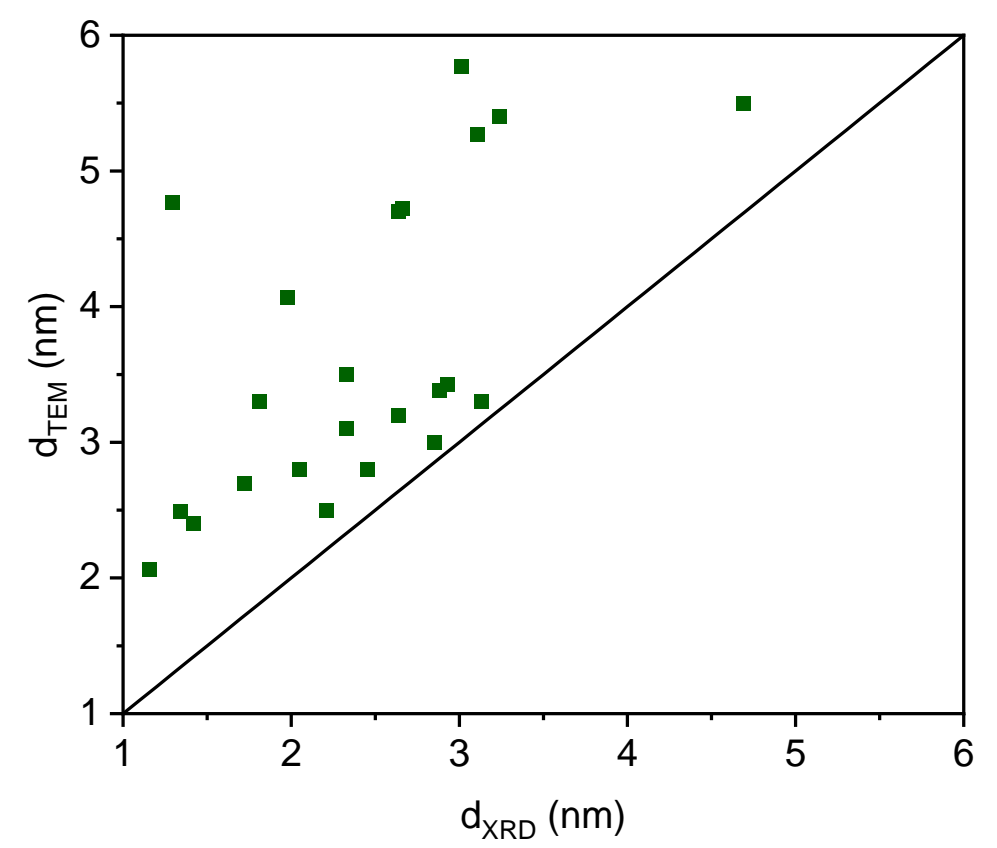

Figure S6: Plot showing $\mathrm{d}_{\mathrm{TEM}} \mathrm{vs}$. $\mathrm{d}_{\mathrm{XRD}}$, where the line is $\mathrm{d}_{\mathrm{TEM}}=\mathrm{d}_{\mathrm{XRD}}$.

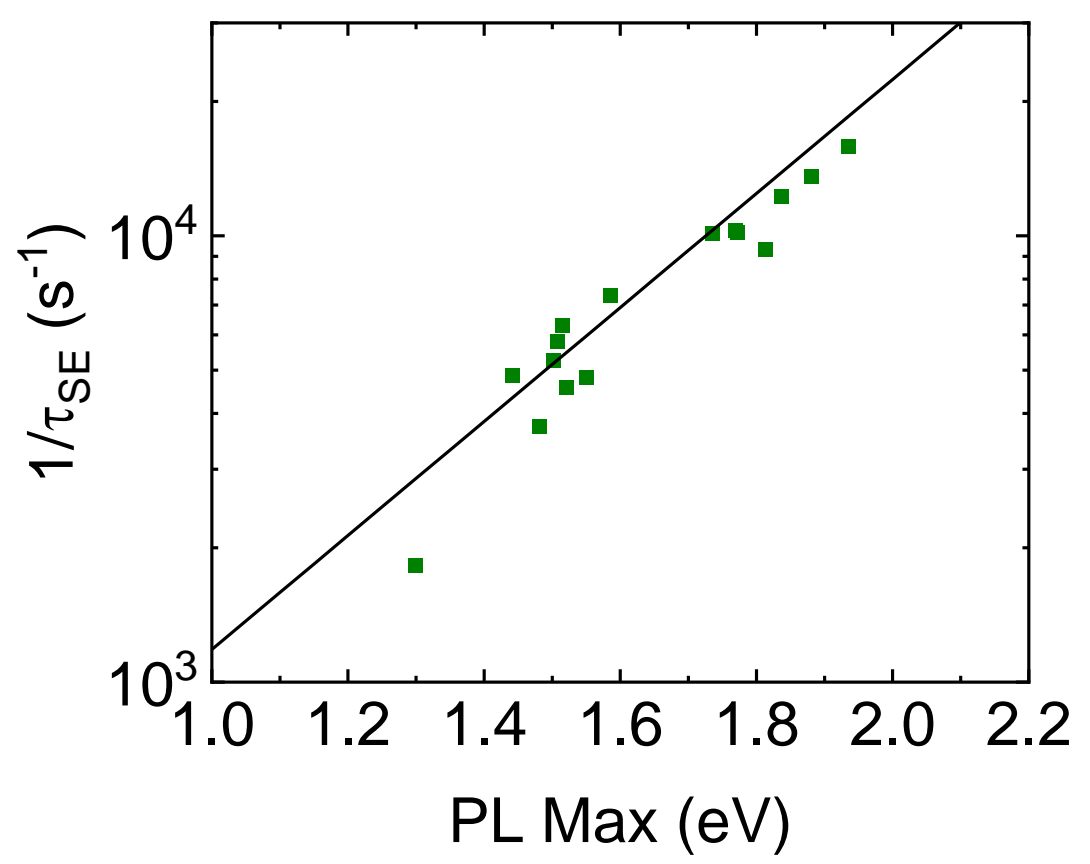

Figure S7: A plot of the maximum photoluminescence energy against the lifetimes for all SiQDs in this study. A clear trend is observed that can be fit to the equation $\frac{1}{\tau_{S E}}=A * e^{E / E_{0}},{ }^{2-4}$ where A $=62.5 \mathrm{~s}^{-1}$ and $\mathrm{E}_{0}=0.34 \mathrm{eV}$ (the black line). 

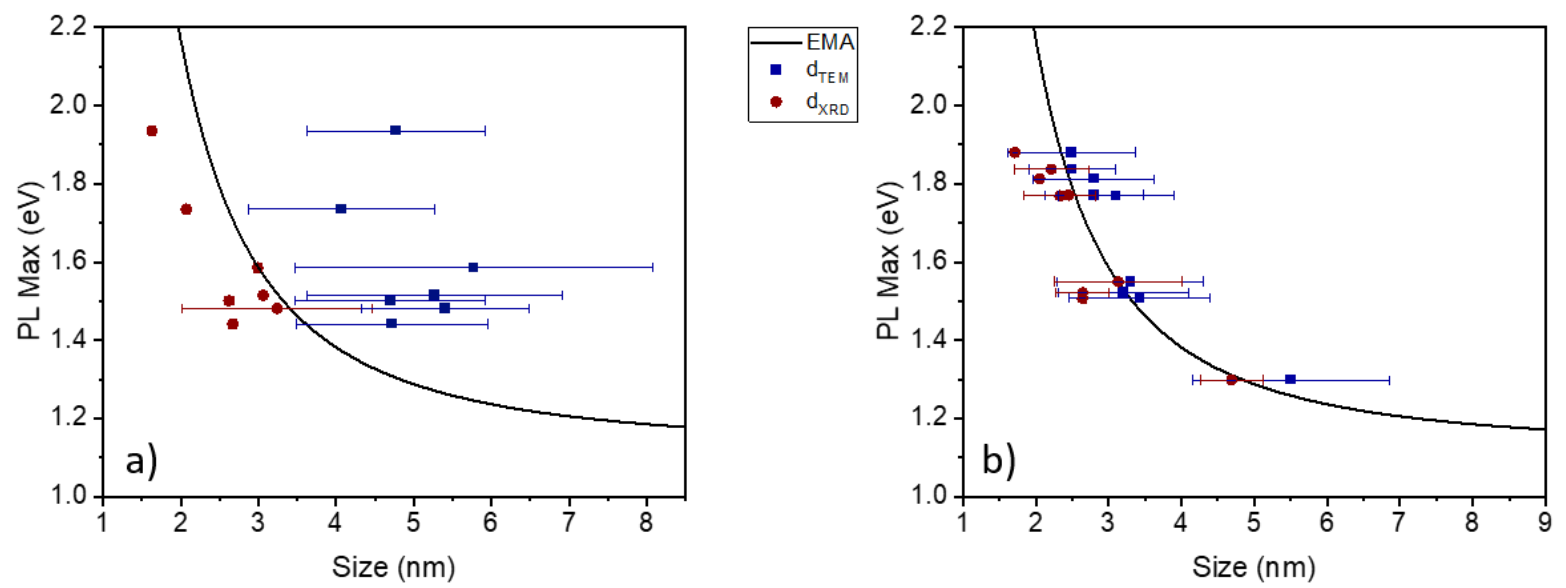

Figure S8: A comparison of the relationship between PL energy with $\mathrm{d}_{\mathrm{TEM}}$ (blue squares; standard deviation shown in blue error bars) and $\mathrm{d}_{\mathrm{XRD}}$ (red circles; fit errors shown in red error bars) for SiQDs with a thick amorphous layer ( $>2 \mathrm{~nm}$; a) and a thin amorphous layer $(<0.8 \mathrm{~nm}$; b). The solid black line in a) and b) represents the EMA as predicted using $E_{g}(r)=1.12+$ $4.19 / r^{2}$.

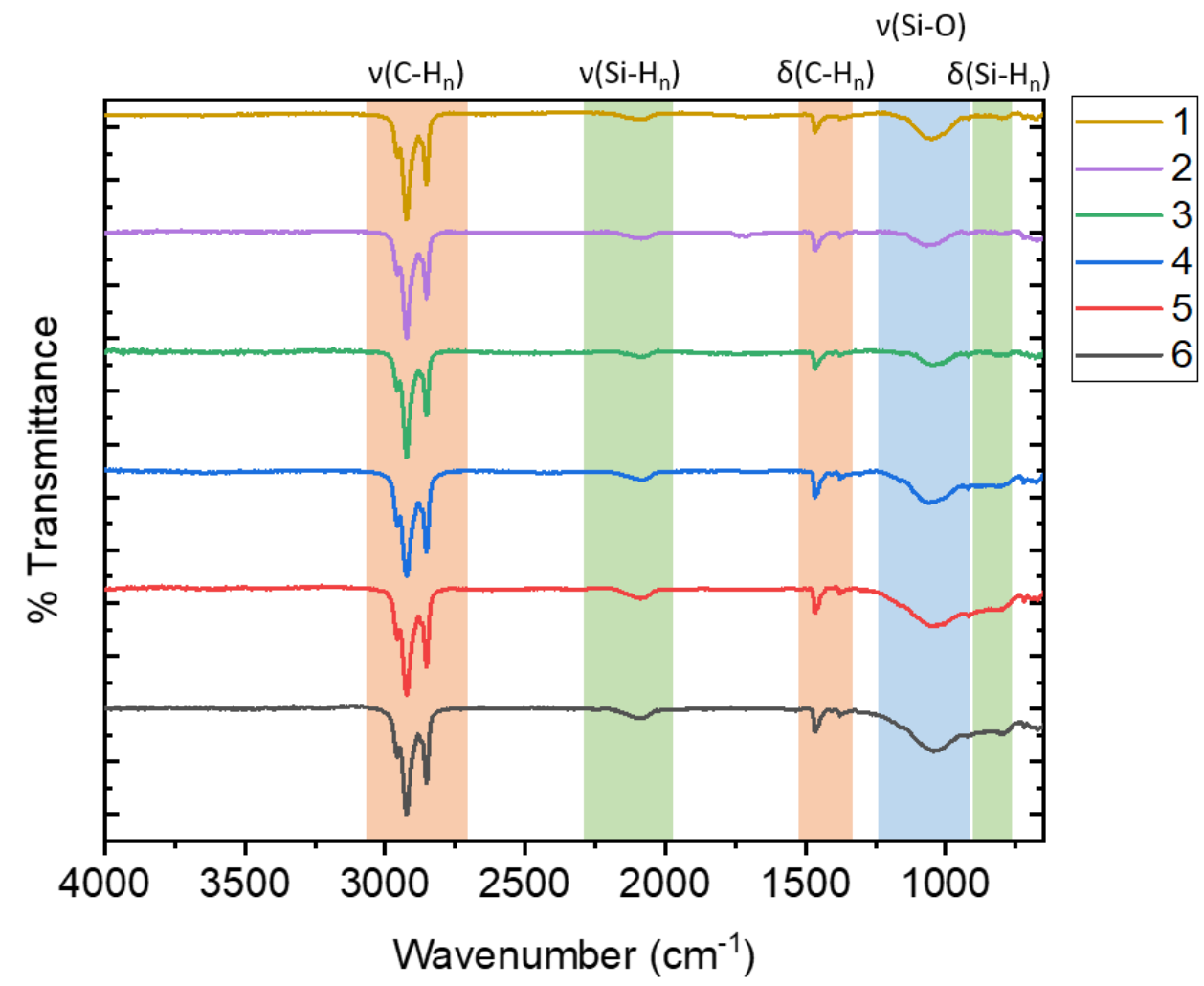

Figure S9: Representative FTIR data showing samples 1 (gold), 2 (purple), 3 (green), 4 (blue), 5 (red), 6 (black). Sample numbers refer to numbers in Table S1. 

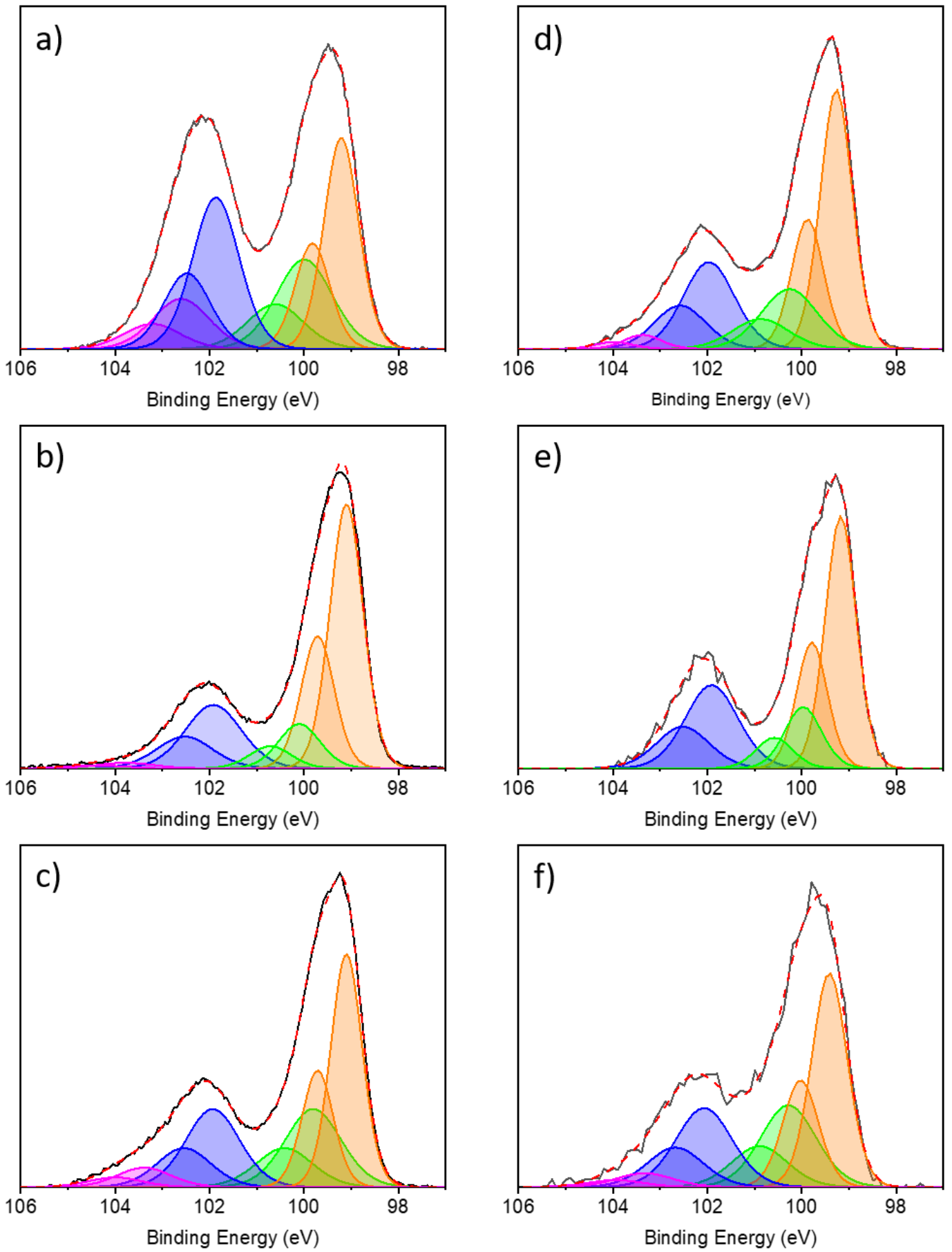

Figure S10: Representative Si 2p XPS data for samples a) 1, b) 2, c) 3, d) 4, e) 5, and f) 6. The oranges peaks represent $\mathrm{Si}(0)$; green, $\mathrm{Si}(\mathrm{I})$; blue, $\mathrm{Si}(\mathrm{III})$; and magenta, $\mathrm{Si}(\mathrm{IV})$. The black solid line shows the experimental data and the red dashed line represents the fitting envelope. Sample numbers refer to numbers in Table $\mathrm{S} 1$. 

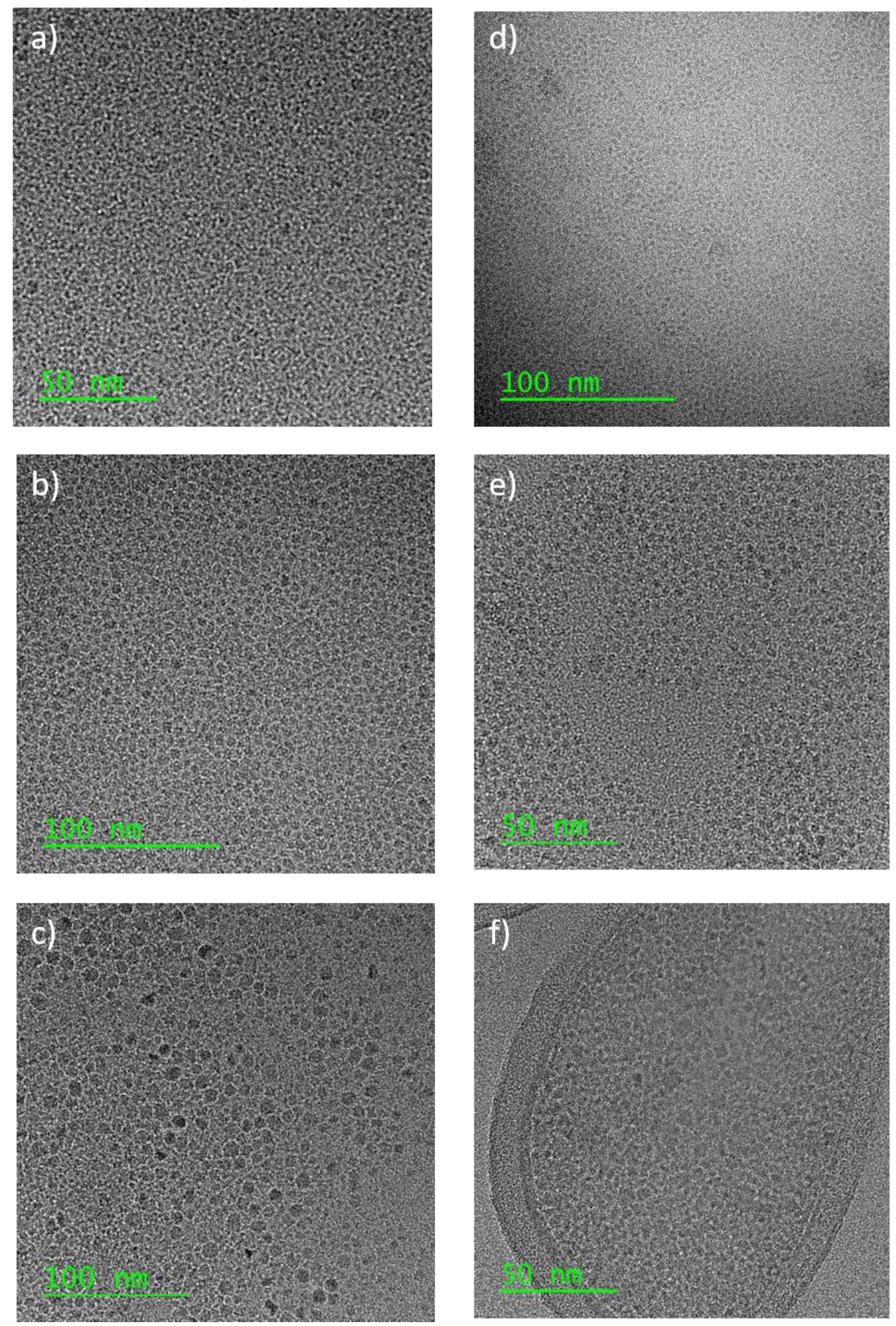

Figure S11: Representative bright field TEM a) 1, b) 2, c) 3, d) 4, e) 5, and f) 6. Sample numbers refer to numbers in Table S1. 
a)

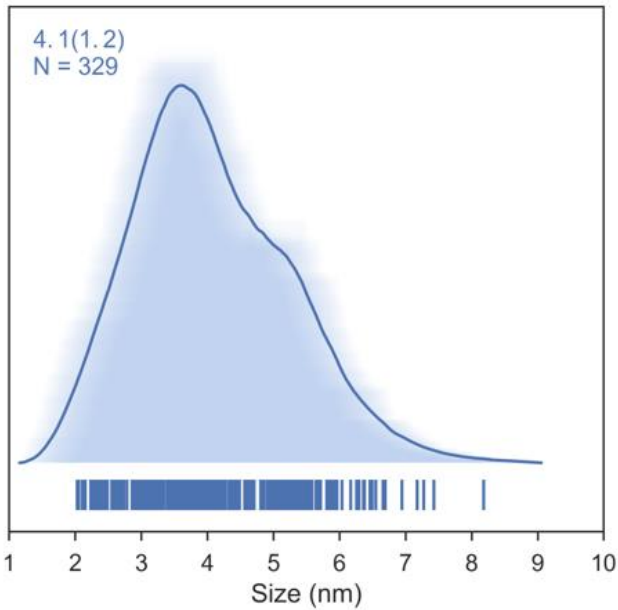

b)

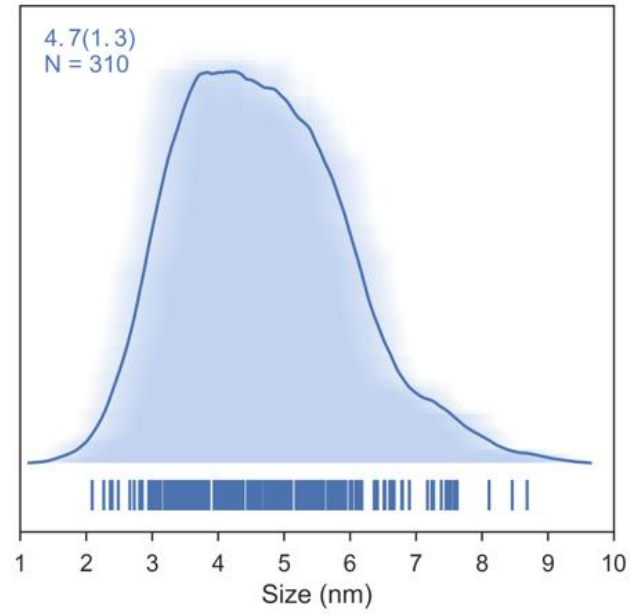

c)

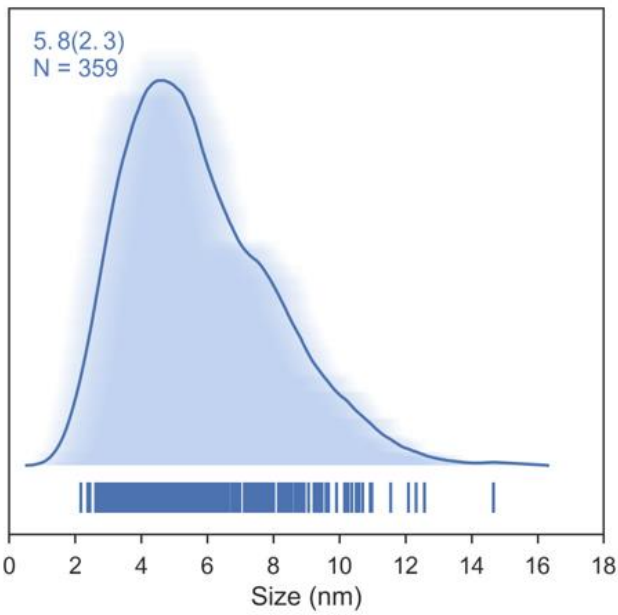

d)

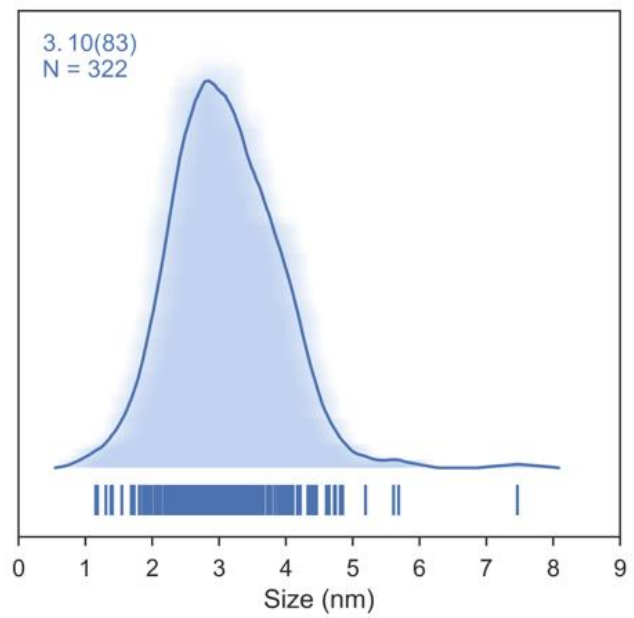

e)

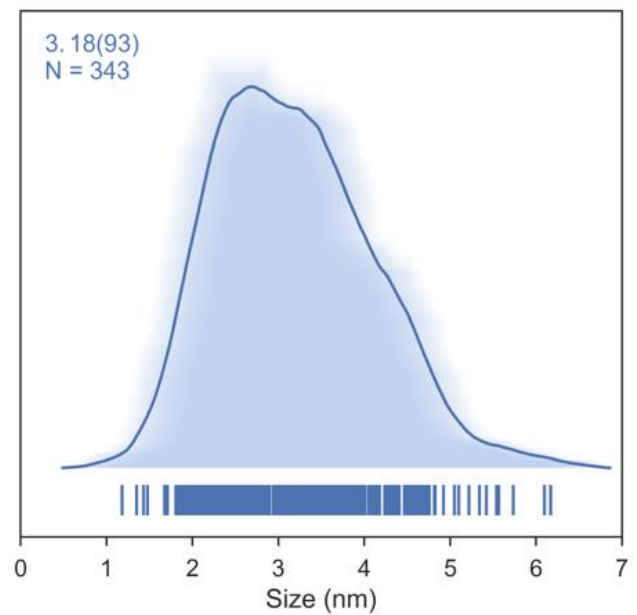

f)

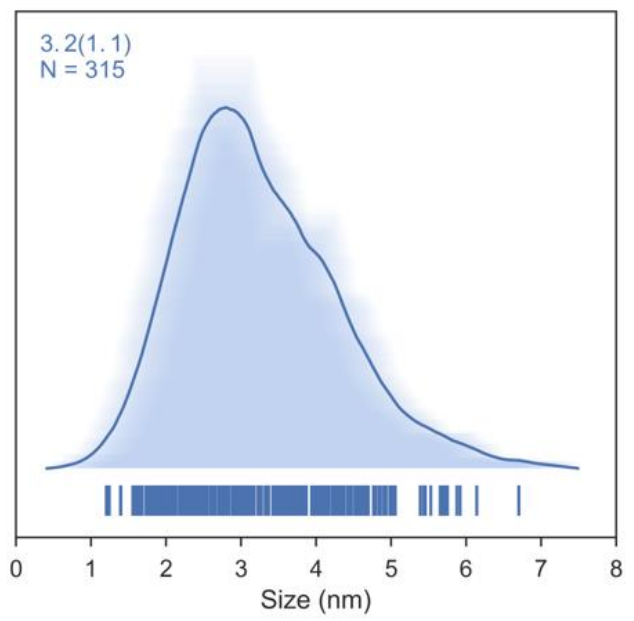

Figure S12: Average shifted histograms for SiNCs counted from TEM/STEM images for samples a)1, b) 2, c) 3, d) 4, e) 5, and f) 6. Sample numbers refer to numbers in Table $S 1$. 


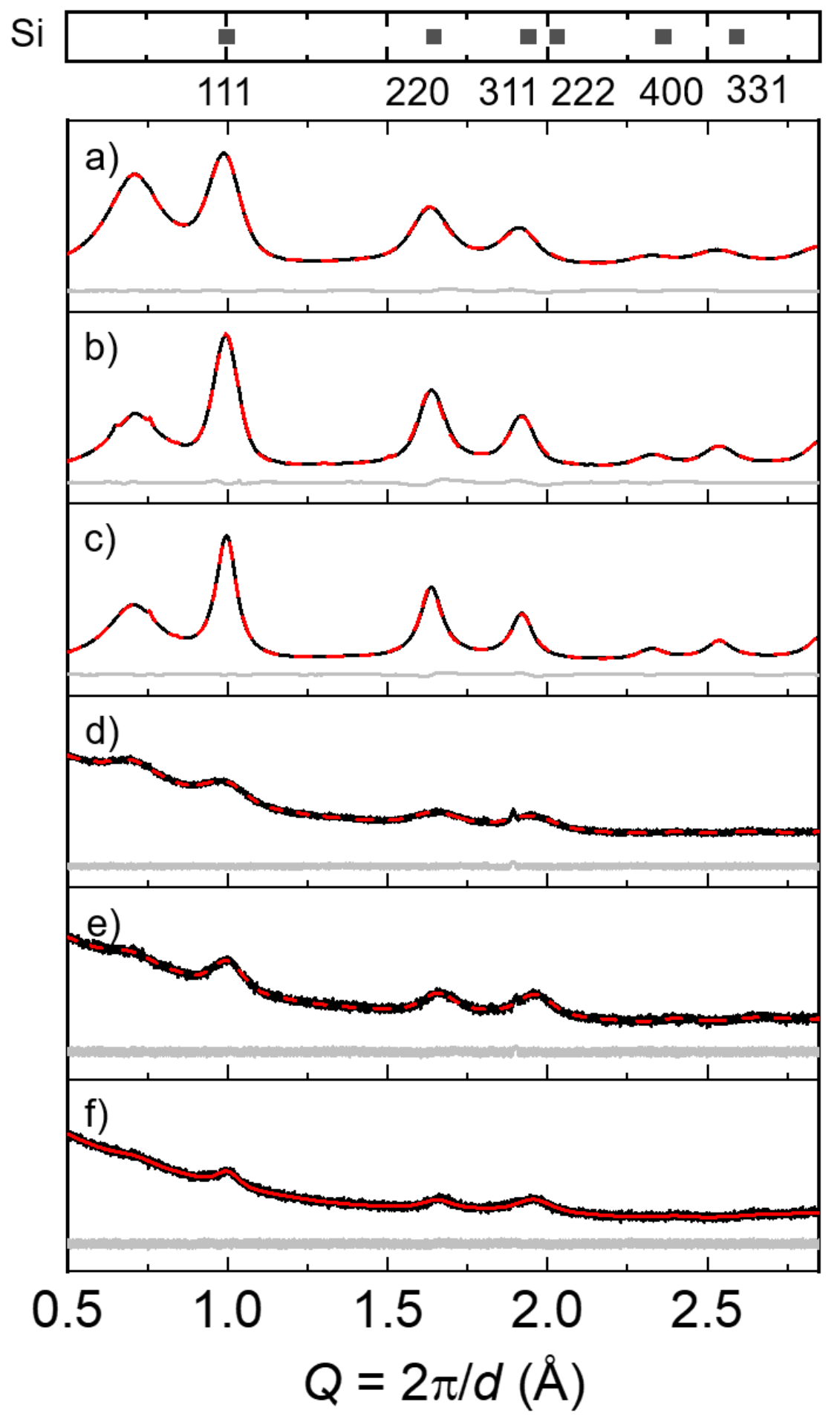

Figure S13: X-ray diffraction powder patterns for samples a) 1, b) 2, c) 3, d) 4, e) 5, and f) 6 . The black trace is the experimental data, the red is the fit and the grey is the difference. Samples a-c were collected at the Canadian Light Source synchrotron facility $(\lambda=0.6891)$ and samples $d-f$ was collected on the Rigaku Ultima with a $\mathrm{Cu}-\mathrm{K} \alpha$ source. Sample numbers refer to numbers in Table S1. 
Table S1: A summary of experimental parameters and results for all samples

\begin{tabular}{|c|c|c|c|c|c|c|c|}
\hline Sample \# & $\begin{array}{c}\text { Anneal } \mathrm{T}^{\mathrm{a}} \\
\left({ }^{\circ} \mathrm{C}\right)\end{array}$ & $\begin{array}{l}\text { Etch time }{ }^{b} \\
\text { (min) }\end{array}$ & $\begin{array}{l}d_{\text {TEM }} \\
(n m)\end{array}$ & $\begin{array}{l}d_{\text {XRD }} \\
(n m)\end{array}$ & $\begin{array}{c}\text { PL Max } \\
\text { (nm) }\end{array}$ & $\begin{array}{l}\text { Lifetime }^{c} \\
(\mu \mathrm{s})\end{array}$ & $\begin{array}{l}\mathrm{QE}^{\mathrm{d}} \\
(\%)\end{array}$ \\
\hline 1300-SiQD & 1300 & $240^{\mathrm{g}}$ & 5.5 & 4.69 & 955 & 546.8 & N/A \\
\hline 1200-SiQD ${ }^{f}$ & 1200 & 60 & 5.4 & 3.24 & 837 & 266.6 & 19.7 \\
\hline $1^{f}$ & 1200 & 90 & 4.1 & 2.07 & 715 & 98.8 & 21.5 \\
\hline $2^{f}$ & 1200 & 60 & 4.7 & 2.62 & 826 & 190.4 & 33.6 \\
\hline $3^{f}$ & 1300 & 135 & 5.8 & 2.99 & 782 & 135.7 & 19.0 \\
\hline $4^{e}$ & 1200 & 165 & 3.1 & 2.33 & 701 & 97.0 & 9.4 \\
\hline $5^{e}$ & 1200 & 75 & 3.2 & 2.64 & 815 & 218.7 & 20 \\
\hline $6^{e}$ & 1300 & 240 & 3.3 & 3.13 & 800 & 208.1 & 14.9 \\
\hline $7^{e}$ & 1100 & 30 & 2.8 & 1.71 & 659 & 73.6 & 8.8 \\
\hline $8^{e}$ & 1200 & 60 & 3.4 & 2.64 & 823 & 172.1 & 33.0 \\
\hline $9^{e}$ & 1200 & 130 & 2.5 & 2.21 & 675 & 81.6 & 7.3 \\
\hline $10^{e}$ & 1200 & 120 & 2.8 & 2.45 & 700 & 98.5 & 10.1 \\
\hline $11^{\mathrm{e}}$ & 1100 & 45 & 2.8 & 2.05 & 684 & 107.3 & 4.3 \\
\hline $12^{f}$ & 1300 & 210 & 5.3 & 3.06 & 819 & 159.0 & 15.6 \\
\hline $13^{f}$ & 1200 & 105 & 4.8 & 1.63 & 641 & 63.1 & 10.0 \\
\hline $14^{f}$ & 1200 & 40 & 4.7 & 2.67 & 860 & 205.7 & 24.5 \\
\hline \\
\hline \multicolumn{8}{|c|}{ b Time used for HF etching } \\
\hline \multicolumn{8}{|c|}{ c Mean lifetimes were calculated as described above. } \\
\hline \multicolumn{8}{|c|}{ d Quantum efficiency } \\
\hline \multicolumn{8}{|c|}{ e "Thin" amorphous shell samples (Figure $4 \mathrm{~b}$ and $\mathrm{d}$ ). } \\
\hline \multicolumn{8}{|c|}{ f "Thick" amorphous shell samples (Figure $4 \mathrm{a}$ and c). } \\
\hline $5 \mathrm{~mL}$ of $49 \%$ & IF added ha & way through & & & & & \\
\hline
\end{tabular}

Table S2: Lifetime fitting parameters for 1200-SiQDs and 1300-QDs

\begin{tabular}{|l|l|l|l|}
\hline & $A$ & $\beta$ & $D c$ \\
\hline $1200-$ SiQDs & 1.031 & 0.8608 & 0.00091 \\
\hline $1300-$ SiQDs & 1.024 & 0.8346 & 0.0053 \\
\hline
\end{tabular}

\section{References}

1. Jakob, M.; Aissiou, A.; Morrish, W.; Marsiglio, F.; Islam, M.; Kartouzian, A.; Meldrum, A., Reappraising the Luminescence Lifetime Distributions in Silicon Nanocrystals. Nanoscale Res. Lett. 2018, 13 (1), 383.

2. $\quad$ Delerue, C.; Allan, G.; Reynaud, C.; Guillois, O.; Ledoux, G.; Huisken, F., Multiexponential photoluminescence decay in indirect-gap semiconductor nanocrystals. Phys. Rev. B 2006, 73 (23), 235318. 
3. Miller, J. B.; Van Sickle, A. R.; Anthony, R. J.; Kroll, D. M.; Kortshagen, U. R.; Hobbie, E. K., Ensemble Brightening and Enhanced Quantum Yield in Size-Purified Silicon Nanocrystals. ACS Nano 2012, 6 (8), 7389-7396.

4. Huisken, F.; Ledoux, G.; Guillois, G; Reynaud, C., Light-Emitting Silicon Nanocrystals from Laser Pyrolysis. Adv. Mater. 2002, 14 (24), 1861-1865. 
